\title{
PATRícIa ZamberLAN dOS SANTOS
}

\section{Composição corporal como indicadora de}

\author{
prognóstico em crianças e adolescentes
}

\author{
gravemente doentes
}

Tese apresentada à Faculdade de Medicina da Universidade de São Paulo para obtenção do título de Doutora em Ciências

Programa de Pediatria

Orientador: Prof. Dr. Artur Figueiredo Delgado 


\title{
PATRícIa ZamberLAN dOS SANTOS
}

\section{Composição corporal como indicadora de}

\author{
prognóstico em crianças e adolescentes
}

\author{
gravemente doentes
}

Tese apresentada à Faculdade de Medicina da Universidade de São Paulo para obtenção do título de Doutora em Ciências

Programa de Pediatria

Orientador: Prof. Dr. Artur Figueiredo Delgado

\section{SÃO PAULO}

2019 


\section{Dados Internacionais de Catalogação na Publicaçäo (CIP)}

Preparada pela Biblioteca da

Faculdade de Medicina da Universidade de São Paulo

Greproduçäo autorizada pelo autor

Santos, Patr1c1a Zamberlan dos

Compos1çlo corporal como 1ndicadora de progn6stico

em crianças e adolescentes gravemente doentes /

Patric1a Zamberlan dos Santos. -- Såo Paulo, 2019.

Tese (doutorado) --Paculdade de Medicina da

Un1vergidade de såo paulo.

Programa de Pediatria.

Or1entador: Artur P1gue1redo Delgado.

Descr1tores: 1.Aval1açăo nutricional

2. Mortal1dade 3. Un1dades de terap1a 1ntens1va

pediatr1ca 4.Progn6atico 5.Compos1çăo corporal

6. Angulo de fase

USP/FM/DBD-053/19

Responsável: Erinalva da Conceição Batista, CRB-8 6755 


\section{Dedicatóría}

Aos meus pais, Paulo (in memorian) e Tereza, por todo o sacrifício para que eu pudesse estudar, e pelo exemplo de vida baseado na ética, honestidade e amor ao próximo.

À minha família, que está comigo nas alegrias e nas dificuldades.

À minha irmã Renata pela amizade e cumplicidade durante toda a vida.

À minha amiga de todas as vidas, Maria de Fátima Henriques Vieira, pelos momentos inesquecíveis de convivência... 


\section{AGRADECIMENTOS}

Ao Prof. Dr. Artur Figueiredo Delgado, meu grande amigo e orientador, pela parceria, incentivo e constante apoio científico.

Ao Dr. Ulysses Doria Filho, pela realização da análise estatística.

Às nutricionistas do Instituto da Criança, pela inestimável ajuda durante todo o estudo, e em especial à Karina Helena Canton Viani pelo providencial auxílio na análise estatística final e à Vanessa da Cunha Oliveira pela elaboração das figuras.

Às equipes de enfermagem e fisioterapia da Unidade de Terapia Intensiva Pediátrica, pelo valioso auxílio para a precisa coleta dos dados.

Ao Prof. Rubens Feferbaum, pelos anos de trabalho juntos.

Ao querido Dr. Ary Lopes Cardoso, por todo o ensinamento.

À Dra Rosana Tumas pela amizade.

Aos professores Werther Brunow de Carvalho e Uenis Tannuri pelo constante incentivo.

À bibliotecária Mariza Umetsu, por todo o apoio na estruturação da bibliografia e pela amizade.

Às crianças que compuseram esta casuística. 
"It's a beautiful day to save lives".

Dr.Derek Shepherd 
Esta tese está de acordo com as seguintes normas, em vigor no momento desta publicação:

Referências: adaptado de International Committee of Medical Journals Editors (Vancouver)

Universidade de São Paulo. Faculdade de Medicina. Serviço de Biblioteca e Documentação. Guia de apresentação de dissertações, teses e monografias. Elaborado por Annelise Carneiro da Cunha, Maria Julia de A. L. Freddi, Maria F. Crestana, Marinalva de Souza Aragão, Suely Campos Cardoso, Valéria Vilhena. 3aㅡ ed. São Paulo: Divisão de Biblioteca e Documentação; 2011.

Abreviaturas dos títulos dos periódicos de acordo com List of Journals Indexed in Index Medicus. 


\section{SUMÁRIO}

Lista de abreviaturas e siglas

Lista de figuras

Lista de tabelas

Lista de gráficos

Resumo

Summary

\section{INTRODUÇÃO}

1.1 Subnutrição em crianças gravemente doentes ....................................... 02

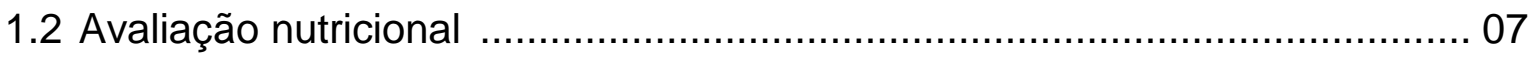

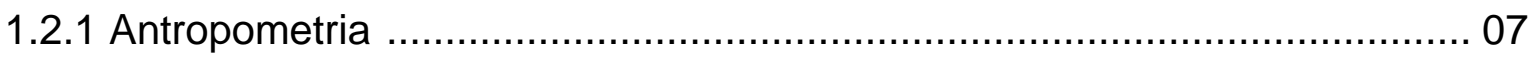

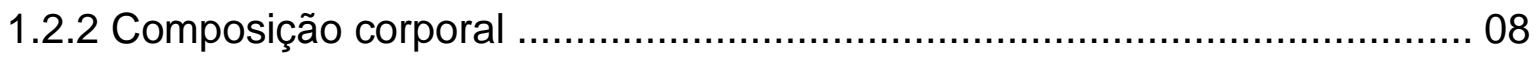

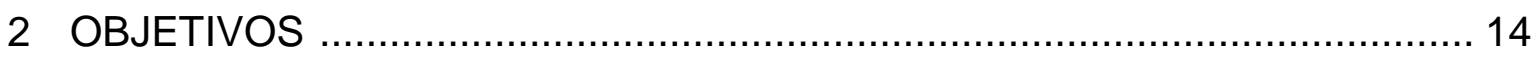

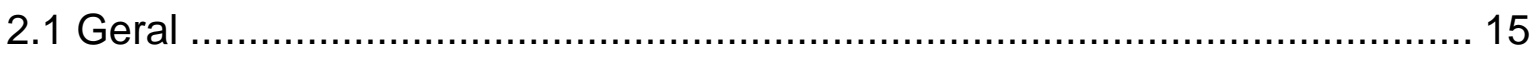

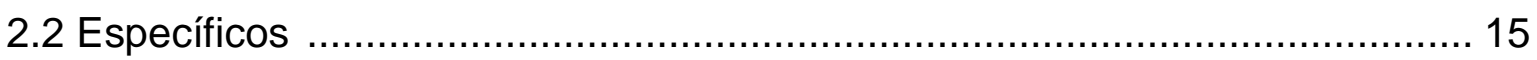

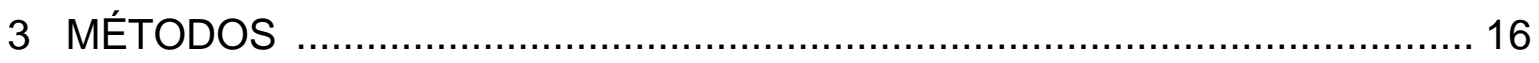

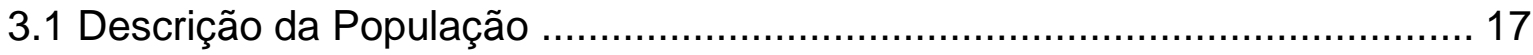

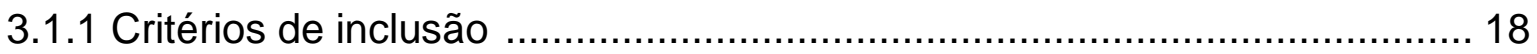

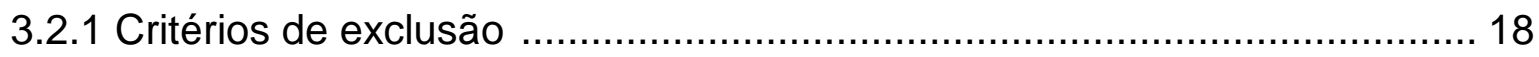

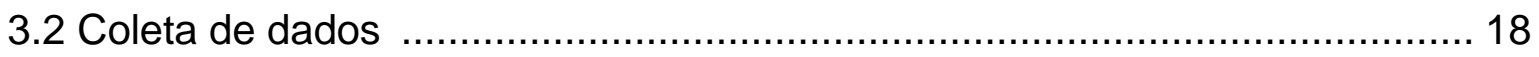

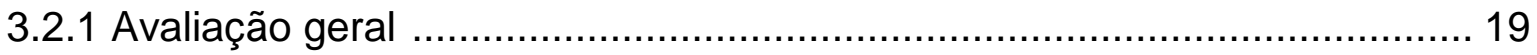

3.2.2 Avaliação nutricional antropométrica ................................................ 19

3.2.3 Avaliação da composição corporal .................................................... 21

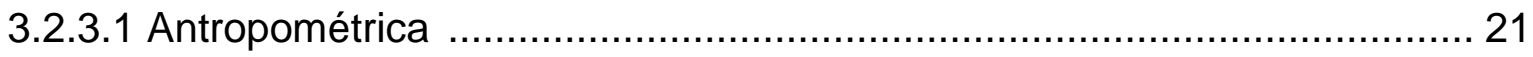

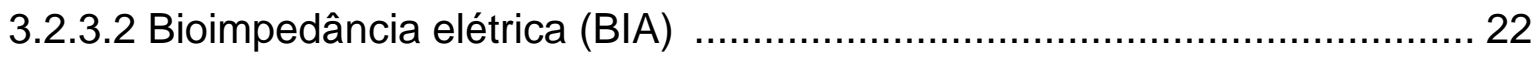

3.2.4 Avaliação de risco e gravidade ..................................................... 22

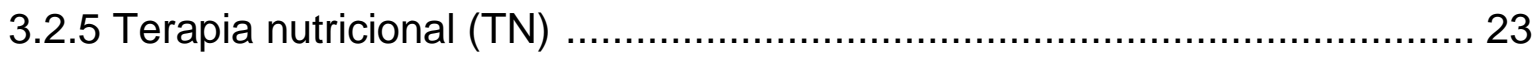

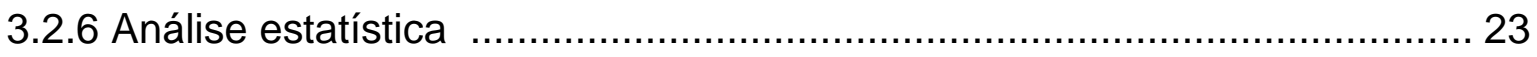

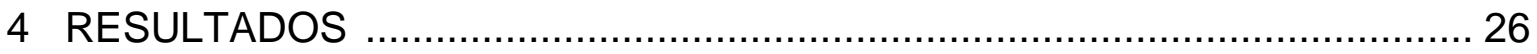

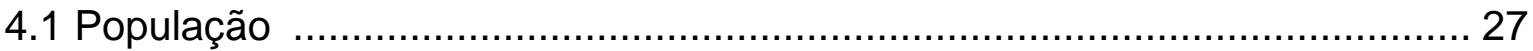

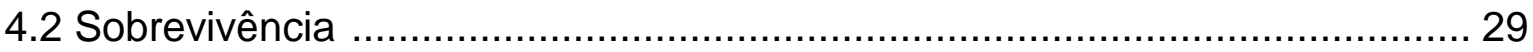

4.3 Tempo de internação e indicadores de composição corporal ....................... 33

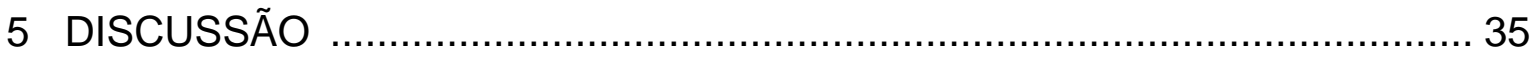

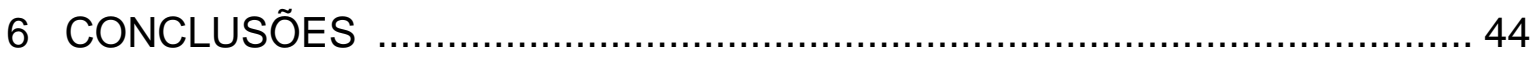




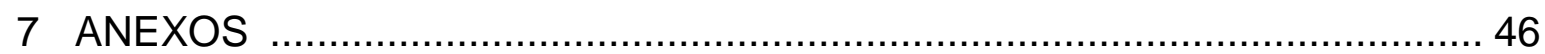

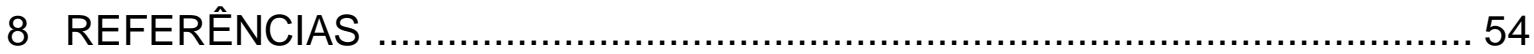




\section{LISTA DE ABREVIATURAS E SIGLAS}

\begin{tabular}{|c|c|}
\hline ACT & Água corporal total \\
\hline $\mathrm{AF}$ & Ângulo de fase \\
\hline AMB & Área muscular do braço \\
\hline ASPEN & American Society of Parenteral and Enteral Nutrition \\
\hline$A \cup C$ & Área sob a curva \\
\hline $\mathrm{BIA}$ & Bioimpedância elétrica \\
\hline CAPPesq & Comissão de Análise de Projetos de Pesquisa \\
\hline $\mathrm{CB}$ & Circunferência do braço \\
\hline COVISA & $\begin{array}{l}\text { Coordenadoria de Vigilância em Saúde da Secretaria } \\
\text { Municipal de Saúde de São Paulo }\end{array}$ \\
\hline DCT & Dobra cutânea triciptal \\
\hline DEXA & Dual-energy $X$-ray absorptiometry \\
\hline$E / l$ & Estatura para idade \\
\hline EMTN & Equipe Multiprofissional de Terapia Nutricional \\
\hline FA-UTI & Fraqueza adquirida na UTI \\
\hline HC-FMUSP & $\begin{array}{l}\text { Hospital das Clínicas da Faculdade de Medicina da } \\
\text { Universidade de São Paulo }\end{array}$ \\
\hline $\mathrm{HR}$ & Hazard ratio \\
\hline I & Idade \\
\hline IC & Intervalo de confiança \\
\hline $\mathrm{IL}-1 \beta$ & Interleucina-1 $\beta$ \\
\hline
\end{tabular}




$\begin{array}{ll}\text { IL-6 } & \text { Interleucina-6 } \\ \text { IMC } & \text { Índice de massa corpórea } \\ \text { IMC/I } & \text { Índice de massa corpórea para a idade } \\ \text { MG } & \text { Massa gorda } \\ \text { MLG } & \text { Massa livre de gordura } \\ \text { OR } & \text { Odds ratio } \\ \text { P/E } & \text { Peso para estatura } \\ \text { P/I } & \text { Peso para idade } \\ \text { PIM } & \text { Paediatric Index of Mortality } \\ \text { R } & \text { Resistência } \\ \text { ROC } & \text { Receiver Operating Characteristic } \\ \text { TCLE } & \text { Termo de consentimento livre e esclarecido } \\ \text { TN } & \text { Terapia nutricional } \\ \text { TNF- } \alpha & \text { Fator de necrose tumoral alfa } \\ \text { UTI } & \text { Unidade de Terapia Intensiva } \\ \text { UTIP } & \text { Unidade de Terapia Intensiva Pediátrica } \\ \text { Xc } & \text { Reactância } \\ \text { Z } & \text { escore }\end{array}$




\section{LISTA DE FIGURAS}

Figura 1 - Resposta metabólica ao estresse no paciente

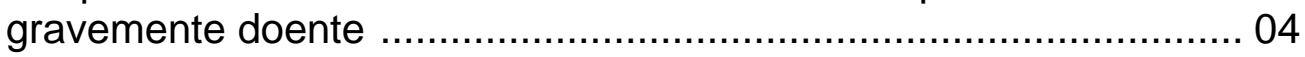

Figura 2 - Demonstração da derivação gráfica do AF …………………….... 12

Figura 3 - Mensuração da CB ............................................................... 21

Figura 4 - Colocação dos eletrodos para o exame de BIA ............................... 22

Figura 5 - Metodologia do estudo ................................................................... 25

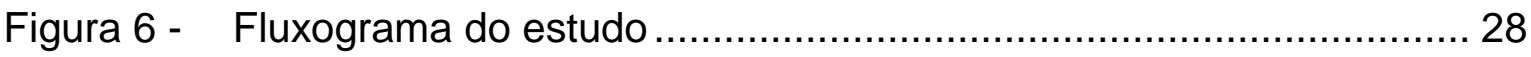




\section{LISTA DE TABELAS}

Tabela 1 - Estado nutricional de crianças e adolescentes admitidos na UTIP

Tabela 2 - Estimativa de mortalidade para crianças e adolescentes gravemente doentes a partir de análise ajustada do modelo de regressão de Cox para AF

Tabela 3 - Estimativa de mortalidade para crianças e adolescentes gravemente doentes a partir de análise ajustada do modelo de regressão de Cox para CB

Tabela 4 - Hazard ratio de tempo de internação em crianças com $\mathrm{AF} \leq 2,8^{\circ}$ e $\mathrm{pCB} \leq 5$ 


\section{LISTA DE GRÁFICOS}

Gráfico 1 - Curva ROC de AF vs mortalidade em crianças e adolescentes na UTIP

Gráfico 2 - Curva de sobrevivência Kaplan-Meier para crianças e adolescentes gravemente doentes usando o cutoff de AF obtido pela BIA

Gráfico 3 - Curva de sobrevivência Kaplan-Meier para crianças e adolescentes gravemente doentes usando o cutoff de $\mathrm{pCB}$

Gráfico 4 - Curva de Kaplan-Meier da associação de AF e tempo de internação em crianças e adolescentes gravemente doentes

Gráfico 5 - Curva de Kaplan-Meier da associação de CB e tempo de internação em crianças e adolescentes gravemente doentes 


\section{RESUMO}

Santos PZ. Composição corporal como indicadora de prognóstico em crianças e adolescentes gravemente doentes [tese]. São Paulo: Faculdade de Medicina, Universidade de São Paulo; 2019.

A subnutrição é uma condição muito prevalente em crianças gravemente doentes em todo o mundo, e está associada a maior morbimortalidade, incluindo maior risco de infecções devido à desordem imunológica transitória, cicatrização inadequada, função intestinal reduzida, maior dependência de ventilação mecânica e tempo de internação hospitalar. Estudos atuais têm proposto que uma intervenção nutricional precoce direcionada pela avaliação nutricional pode prevenir ou minimizar as complicações da subnutrição. $O$ estresse promove uma resposta inflamatória aguda mediada por citocinas, que resulta em aumento do metabolismo basal e da excreção nitrogenada, levando a grande perda muscular e alterações da composição corpórea. Por essa razão, a inclusão da avaliação da composição corporal torna-se importante na avaliação desses pacientes gravemente doentes, uma vez que além do aspecto nutricional, a composição corporal parece ser capaz de predizer prognósticos clínicos. Existem várias técnicas para avaliar a composição corporal, como as medidas do braço e a bioimpedância elétrica (BIA). Dentre os parâmetros da BIA, o ângulo de fase (AF), que representa indiretamente a massa livre de gordura (MLG), é o mais clinicamente estabelecido, uma vez que tem mostrado grande capacidade de predizer desfechos em uma ampla variedade de situações clínicas. Dada a importância da análise da composição corporal como adjunta da avaliação nutricional e como possível indicadora de prognóstico de morbimortalidade em diversas situações clínicas, faz-se importante a realização de estudos que comprovem sua efetividade, especialmente em crianças gravemente doentes, onde os dados ainda são escassos. Objetivo: $O$ objetivo deste estudo foi testar a associação do estado nutricional na admissão avaliado por indicadores de composição corporal circunferência do braço (CB) e AF - com tempo de internação e mortalidade em até 30 dias em crianças e adolescentes gravemente doentes na unidade de terapia intensiva pediátrica (UTIP). Métodos: Foram coletados dados antropométricos e demográficos de crianças de 2 meses a 18 anos internadas na UTI do Instituto da Criança do Hospital das Clínicas da Universidade de São Paulo, no período de um ano. A avaliação nutricional antropométrica foi realizada nas primeiras 24 horas de admissão para caracterizar a população do estudo e incluiu peso, altura ou comprimento e índice de massa corporal (IMC), além da CB. A classificação antropométrica na admissão foi definida pelo escore z (Z) do IMC para a idade (IMC/I), utilizando os valores de referência da Organização Mundial da Saúde (OMS). Para a classificação da CB foram utilizados os percentis propostos por Frisancho. A BIA foi realizada para a 
obtenção do AF. Os pacientes admitidos foram avaliados quanto à gravidade por intermédio do escore "Paediatric Index Mortality" (PIM). Análise estatística descritiva foi utilizada para as variáveis nominais. Uma curva ROC ("Receiver Operating Characteristic") foi construída para analisar a associação do AF com mortalidade em até 30 dias na UTIP e encontrar o melhor cutoff associado a esta mortalidade. As probabilidades de sobrevivência foram estimadas pelo método de Kaplan-Meier e comparadas pelo teste de log-rank, e a taxa de falha foi determinada pelo modelo de regressão de Cox. A análise de tempo para o evento (curva de Kaplan-Meier) foi utilizada para avaliar a influência dos indicadores de composição corporal no tempo de internação na UTIP, seguida do teste de log-rank para verificar diferença significativa entre as curvas. Resultados: Foram avaliadas 247 crianças com mediana de idade de 4,8 anos e cuja principal causa de internação foi sepse. A mediana do tempo de internação na UTIP e do PIM foi de 4,0 e 2,7, respectivamente. Houve predomínio de pacientes eutróficos de acordo com o Z IMC/I (49,8\%), e quase metade dos pacientes $(49,8 \%)$ apresentou depleção da MLG segundo o percentil da $\mathrm{CB}$ (abaixo do percentil 5 ). $\mathrm{O}$ cutoff do $\mathrm{AF}$ associado à mortalidade em crianças gravemente doentes encontrado pela análise da curva ROC foi de $2,8^{\circ} \quad(A \cup C=0,65 ;$ IC95\%: 0,58-0,71), com sensibilidade de $37,1 \%$ e especificidade de $86 \%$. As curvas mostraram maior sobrevivência em pacientes com $\mathrm{AF}>2,8^{\circ}$ quando comparados àqueles com $\mathrm{AF} \leq 2,8^{\circ}$ (53 vs 23 dias, respectivamente; $p<0,0001)$. A análise de Kaplan-Meier de tempo para 0 evento mostrou que as crianças que não morreram e apresentavam menores valores de AF tinham maior probabilidade de permanecerem internadas por mais tempo na UTI (HR: 1,$84 ; p=0,003$ ). Menor sobrevivência também foi observada em pacientes que apresentavam percentis de CB inferior a 5 quando comparados aos com percentil de CB maior do que 5 (26 vs 28 dias; $p$ $<0,03)$. Conclusões: Houve associação do $A F$ e da $C B$ com mortalidade e morbidade em crianças gravemente doentes, sugerindo que esses parâmetros podem ser úteis não apenas para o diagnóstico e monitoramento nutricional, mas também como um indicador adicional na estimativa do prognóstico. $O$ cutoff de AF encontrado $\left(2,8^{\circ}\right)$ parece ser mais apropriado para avaliar a associação com mortalidade nessa população.

Descritores: avaliação nutricional; mortalidade; unidades de terapia intensiva pediátrica; prognóstico; composição corporal; ângulo de fase. 


\section{SUMMARY}

Santos PZ. Body composition as prognosis indicator in critically ill children and adolescents [thesis]. São Paulo: "Faculdade de Medicina, Universidade de São Paulo"; 2019.

Undernutrition is highly prevalent in critically ill children throughout the world and it is associated with increased morbidity and mortality, including a higher risk of infection due to transitory immunological disorder, inadequate woundhealing, reduced gut function, longer dependency on mechanical ventilation and longer hospital stays. Nutritional care studies have proposed that an early intervention targeting nutrition assessment can prevent or minimize the complications of undernutrition. Stress promotes an acute inflammatory response mediated by cytokines resulting in increased basal metabolism and nitrogen excretion leading to major muscle loss and changes in body composition. For this reason the inclusion of body composition assessment becomes important in the evaluation of these patients since in addition to the nutritional aspect, body composition seems to be able to predict clinical prognosis. There are several techniques to assess body composition, such as arm measurements and bioelectrical impedance analysis (BIA). Among the BIA parameters, the phase angle (PA), which indirectly represents fat free mass (FFM), is the most clinically established, since it has shown a strong ability to predict outcomes in a wide variety of clinical situations. Considering the importance of body composition analysis as an adjunctive nutritional assessment and as a possible morbidity and mortality predictor in many clinical situations, it is necessary to conduct studies that demonstrate this ability, especially in critically ill children, since data are still scarce. Objective: The aim of this study was to test the association of nutritional status on admission evaluated by body composition indicators - arm circumference (AC) and PA with pediatric intensive care unit (PICU) length of stay (LOS) and 30-day mortality in critically ill children and adolescents. Methods: Anthropometric and demographic data from children aged 2 months to 18 years admitted to the PICU of Instituto da Criança, Hospital das Clínicas da Universidade de São Paulo during a one-year period were collected. Anthropometric nutritional assessment was performed within 24 hours of admission to characterize the study population. The assessment included weight, height or length, and body mass index (BMI), besides AC. Anthropometric classification on admission was defined by the BMI-for-age $\mathrm{z}$-score using the reference values from the World Health Organization (WHO). CB was classified by percentiles proposed by Frisancho. BIA was performed to obtain PA. Severity was evaluated by scoring the Pediatric Index of Mortality (PIM). Descriptive statistics were reported for nominal variables. Receiver Operating Characteristic $(\mathrm{ROC})$ curve was used to 
analyze the association of PA with 30-day mortality and to find the best cutoff. Survival probabilities were estimated by the Kaplan-Meier method, and they were compared using the log-rank test. The failure rate was estimated according to models of Cox regression. The Kaplan-Meier time-to-event analysis was used to assess the influence of the body composition indicators on the PICU LOS, followed by the log-rank test to verify any significant difference between the curves. Results: We evaluated 247 children with a median age of 4.8 years whose main cause of admission was sepsis. The median PICU LOS was 4 days and median PIM was 2.7. There was a predominance of eutrophic patients according to BMI-for-age z-scores (49.8\%), and almost half of the patients (49.8\%) presented FFM depletion according to the percentile AC (below the 5th percentile). PA cutoff associated with mortality in critically ill children found by the ROC curve analysis was $2.8^{\circ}$ ( $\mathrm{AUC}=0.65 ; 95 \% \mathrm{Cl}$ : $0.58-$ 0.71 ), with a sensitivity of $37.1 \%$ and a specificity of $86 \%$. Survival curves showed higher survival in patients with PA $>2.8^{\circ}$ compared to patients with PA $\leq 2.8^{\circ}$ (53 vs 23 days respectively; $p<0.0001$ ). Kaplan-Meier time-to-event analysis showed that children who did not die and had lower PA values were more likely to remain longer in the PICU (HR: $1.84 ; p=0.003$ ). Lower survival also observed in patients who presented $A C$ values $\leq$ percentile 5 when compared to patients with AC percentile > 5 (26 vs 28 days; $p<0.03$ ). Conclusions: $A C$ and PA were associated with mortality and morbidity in critically ill children, suggesting that these parameters may be useful not only for nutritional diagnosis and monitoring but also for an additional indicator in estimating prognosis. We found that a PA cutoff of $2.8^{\circ}$ seems to be more appropriate to evaluate association with mortality for this population.

Descriptors: nutritional assessment; mortality; intensive care units, pediatric; prognosis; body composition; phase angle. 
1 INTRODUÇÃO 


\subsection{Subnutrição em crianças gravemente doentes}

A subnutrição hospitalar é aquela diagnosticada no hospital - seja na admissão ou durante a internação - e frequentemente está associada a uma doença crônica de base, constituindo um problema de saúde pública, tanto em

países desenvolvidos como naqueles em desenvolvimento. De acordo com a American Society of Parenteral and Enteral Nutrition (ASPEN), a desnutrição adquirida no hospital é um desequilíbrio nutricional que ocorre durante a internação, independentemente de os pacientes apresentarem a condição à admissão ${ }^{1}$. Na grande maioria dos indivíduos hospitalizados, a subnutrição está associada à doença e se desenvolve como consequência de ingestão inadequada de nutrientes e/ou alteração da absorção, administração de fármacos que interferem no processo nutricional, demora na indicação da terapia nutricional (TN), jejum prolongado para procedimentos, alterações metabólicas ou iatrogenias ${ }^{2}$.

Embora seja difícil determinar com precisão sua prevalência devido à falta de critérios diagnósticos padronizados, assim como na população de adultos, em pediatria, a subnutrição hospitalar tem sido amplamente debatida nos últimos anos ${ }^{3-5}$, com estudos recentes mostrando que o número de crianças subnutridas no âmbito hospitalar ainda é elevado. Estudo realizado em um hospital terciário na Bélgica mostrou que 16,6\% das crianças admitidas apresentavam subnutrição aguda ou crônica e que $31,8 \%$ delas perderam peso 
durante a internação ${ }^{6}$. Um número menor, porém significativo, foi encontrado em estudo multicêntrico de 12 países da Europa em 2014, onde a subnutrição foi diagnosticada em $7 \%$ das crianças, sendo que $23 \%$ daquelas que permaneceram por mais de quatro dias internadas apresentaram piora da condição nutricional ${ }^{7}$. Em nosso meio, Pileggi et al..$^{8}$ encontraram $16,1 \%$ de subnutrição entre 292 crianças admitidas em um hospital universitário do estado de São Paulo.

Em crianças gravemente doentes, estudos realizados há mais de 20 anos também já demonstravam que $15 \%$ a $20 \%$ das crianças admitidas em unidades de terapia intensiva pediátrica (UTIP) eram aguda ou cronicamente subnutridas $^{9-11}$, e apesar do avanço tecnológico destas unidades, dados mais recentes registram esta mesma prevalência ${ }^{12-14}$.

O estado nutricional precário resulta em numerosas deficiências funcionais, que levam a desfechos desfavoráveis como aumento da mortalidade, maior tempo de internação, maior incidência de infecções e maior necessidade de ventilação mecânica, com consequente elevação dos custos hospitalares ${ }^{3,15-17}$, especialmente em pacientes gravemente doentes. $O$ estresse ou lesão tecidual promove uma resposta inflamatória aguda mediada por citocinas, especialmente interleucina-6 (IL-6), IL-1 $\beta$ e fator de necrose tumoral alfa (TNF- $\alpha$ ), que resulta em aumento do metabolismo basal e da excreção nitrogenada ${ }^{18}$ (Figura 1). Ocorre proteólise intensa a fim de disponibilizar aminoácidos para a produção de energia e síntese hepática de proteínas de fase aguda; o que acarreta grande perda muscular ${ }^{19-21}$ e alterações da composição corpórea. 


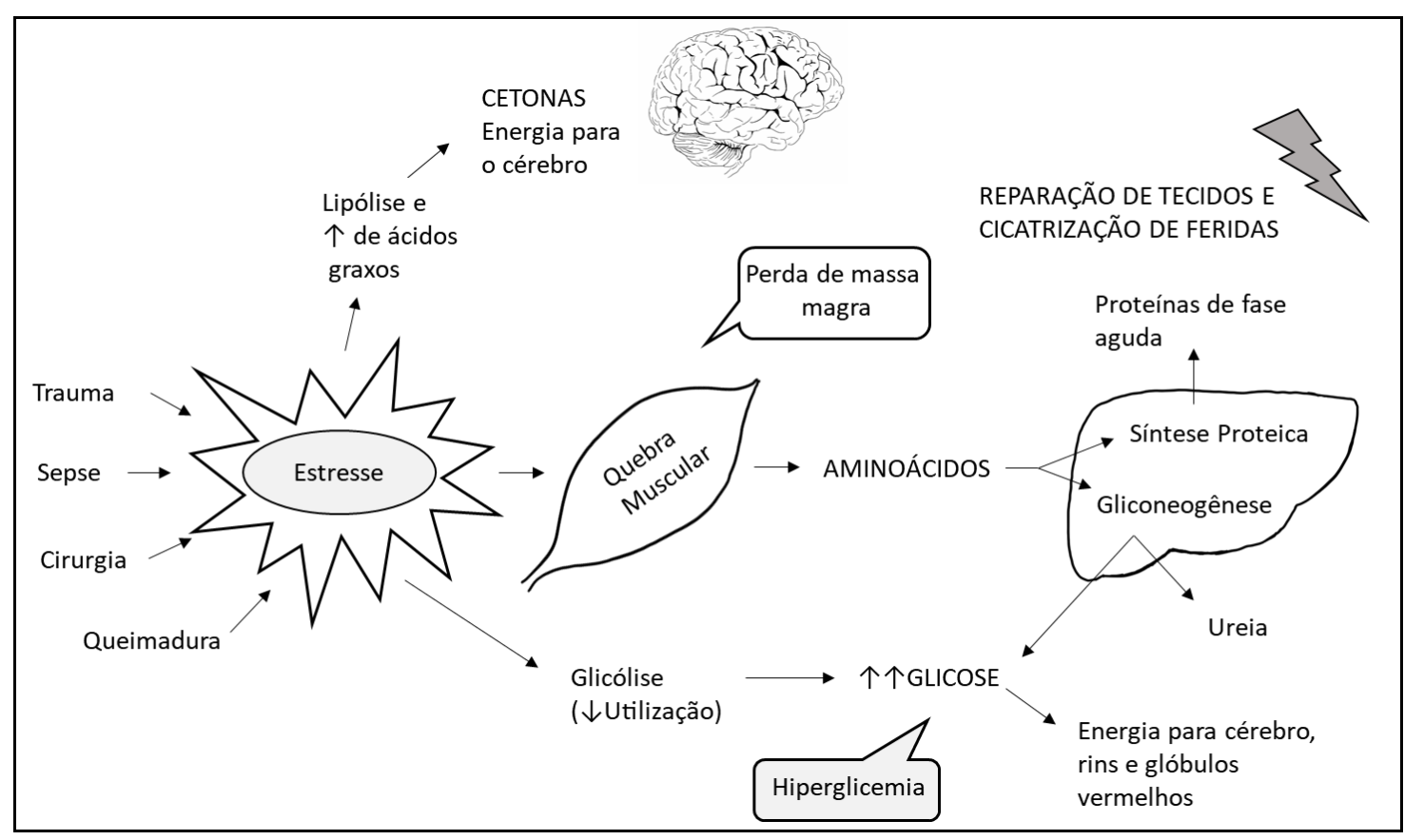

Figura 1 - Resposta metabólica ao estresse no paciente gravemente doente (adaptado de Mehta e Dugan, $2009^{18}$ )

\section{Sarcopenia em pacientes gravemente doentes}

Sarcopenia é definida como a perda de massa e força ou função muscular $^{22}$ e, embora o termo não tenha sido bem descrito em pacientes gravemente doentes, uma condição clínica muito semelhante tem sido relatada nestes pacientes, chamada de fraqueza adquirida na UTI (FA-UTI).

A FA-UTI definida como fraqueza bilateral do membro simétrico, é o resultado de polineuropatia axonal, miopatia, ou frequentemente uma combinação de ambas ${ }^{23,24}$, que afeta principalmente os membros e pode se estender à tetraplegia em casos mais graves ${ }^{25}$. Está associada à fraqueza muscular respiratória, retardo do desmame da ventilação mecânica, maior tempo de internação hospitalar e maior taxa de mortalidade. Estudos têm relatado taxas de prevalência de FA-UTI que variam de 25 a $100 \%$, dependendo dos diferentes critérios de definição ${ }^{23}$. 
A maioria dos fatores de risco para FA-UTI está relacionada à gravidade e duração da resposta inflamatória sistêmica, tempo de internação na UTI, duração da ventilação mecânica e imobilidade; além de idade, sexo (feminino), hiperglicemia, hipoalbuminemia, nutrição parenteral, uso de corticosteroides e bloqueadores neuromusculares. Entretanto, alguns destes fatores de risco como tempo de internação na UTI e duração da ventilação mecânica podem ser consequências da FA-UTI, denotando que há um ciclo vicioso entre estes fatores e a condição propriamente dita ${ }^{26}$.

Garnacho-Montero et al. ${ }^{27}$ encontraram que a polineuropatia desenvolvida em mais de $50 \%$ dos pacientes na UTI foi responsável por maior tempo de ventilação mecânica, bem como período do desmame; além de internação hospitalar e incremento do tempo total de UTI nesta população. Os autores encontraram na análise multivariada que esta polineuropatia estava associada à condição critica como o único fator de risco independente para falha no desmame da ventilação mecânica.

Outro estudo investigando a relação entre FA-UTI, tempo de internação e mortalidade mostrou que pacientes portadores de FA-UTI permaneciam por mais tempo internados tanto na UTI (mediana de 19 vs 10 dias; $p<0,001$ ) como no hospital (mediana de 28 vs 16 dias; $p<0,001$ ) e requeriam um tempo maior de ventilação mecânica (mediana de 12 vs 6 dias; $p<0,001$ ). Além disso, a mortalidade foi maior nestes pacientes (mediana de $31 \%$ vs $6 \% ; p<$ 0,001). O estudo enfatizou que a FA-UTI, bem como a força muscular avaliada, foram fatores independentes associados à mortalidade $(\mathrm{OR} 7,8$; IC 95\%: $2,4-25,3 ; p=0,001$ e OR 4,5; IC 95\%: $1,5-13,6 ; p=0.007$ respectivamente) $)^{28}$. 
Além das consequências em curto prazo, estudos têm demonstrado que a FA-UTI traz impacto à vida dos pacientes em longo prazo. Herridge et al. ${ }^{29}$ demonstraram que a maioria dos pacientes participantes do estudo apresentavam incapacidade funcional um ano após a alta da UTI. Resultados semelhantes foram observados no estudo de Chelluri et $a .^{30}$, onde $57 \%$ dos mais de 800 pacientes avaliados requeriam assistência de cuidadores 12 meses após a alta da UTI.

A sarcopenia é uma importante causa de morbidade e mortalidade em indivíduos idosos, mas ainda pouco conhecida em pacientes gravemente doentes, sejam adultos ou crianças. Existe uma preocupação crescente com esta entidade na UTI, sendo necessários mais estudos que elucidem sua real prevalência e associação com morbimortalidade nestes pacientes. 


\subsection{Avaliação nutricional}

Classicamente, o estado nutricional é avaliado por intermédio tanto de parâmetros subjetivos (triagem nutricional) como de objetivos, que incluem medidas antropométricas e bioquímicas. A avaliação objetiva diagnostica as crianças já subnutridas e a triagem auxilia na identificação daquelas que apresentam risco para a subnutrição, ou seja, risco para a piora da condição nutricional durante a hospitalização ${ }^{31}$. A implementação de uma avaliação nutricional sistematizada é de extrema importância na condução terapêutica do indivíduo hospitalizado, por sinalizar já à internação, aqueles pacientes que se beneficiarão de uma intervenção nutricional precoce e adequada ${ }^{32}$.

\subsubsection{Antropometria}

A antropometria é um componente básico da avaliação nutricional, uma vez que apresenta valores de referência padronizados por faixa etária; além de ser útil para classificação e monitoramento do estado nutricional. Na avaliação de crianças saudáveis (sem comorbidades associadas), os índices antropométricos estão bem estabelecidos, sendo peso/idade (P/l), estatura/idade (E/I), peso/estatura $(P / E)$ e índice de massa corporal/idade (IMC/I) os mais utilizados. No entanto, crianças gravemente doentes são acamadas, o que dificulta a mensuração precisa da estatura. Além disso, devido às alterações metabólicas que ocorrem, especialmente na fase inicial da agressão, comumente apresentam desequilíbrios eletrolíticos, disfunção renal e hepática com edema e ascite, ou massas tumorais que afetam a qualidade da medida de peso. Mensurações imprecisas de peso e altura podem 
comprometer o diagnóstico do estado nutricional, assim como o planejamento da terapia nutricional (TN) e seu monitoramento. Por este motivo, além da antropometria e dos dados laboratoriais, tem sido sugerido incluir na avaliação nutricional destes pacientes, medidas de atividade inflamatória e composição corporal $^{33}$; esta última capaz de fornecer informações precisas sobre os compartimentos corporais, bem como as alterações fisiológicas ou patológicas que neles ocorrem durante o crescimento ou o curso da doença ${ }^{34}$.

\subsubsection{Composição corporal}

Assim como inúmeros indicadores nutricionais ${ }^{35,36}$, a avaliação da composição corpórea vem sendo utilizada como marcador de prognóstico em pacientes gravemente doentes; podendo além de identificar os compartimentos corporais no aspecto nutricional propriamente dito, ser capaz de predizer desfechos clínicos, o que é de extrema importância, uma vez que a identificação precoce de gravidade permite a antecipação de medidas terapêuticas que podem ser decisivas no desfecho do paciente. Comumente, a gravidade de pacientes em UTI é avaliada por escores de prognóstico de mortalidade, que não só determinam o prognóstico e estimam o risco de morte, mas também são úteis para a análise da qualidade do cuidado prestado, bem como para comparação entre diferentes serviços ${ }^{37,38}$.

Em um estudo em que avaliou 240 adultos gravemente doentes em ventilação mecânica, Weijs et al. ${ }^{39}$ mostraram que a redução de massa livre de gordura (MLG), avaliada por tomografia computadorizada, foi um fator de risco para mortalidade independente de sexo e escore de gravidade. Resultados 
similares foram encontrados por Rutten et $a l .{ }^{40}$, onde a redução de um dos compartimentos corporais, MLG ou massa gorda (MG) estava associada a maior taxa de mortalidade entre portadores de doença pulmonar obstrutiva crônica acompanhados prospectivamente por três anos. Em relação a desfechos que não a mortalidade, Pichard et al. ${ }^{41}$ demonstraram que depleção de MLG à admissão hospitalar estava intimamente relacionada à maior tempo de internação.

Várias são as técnicas para avaliar a composição corporal, desde as mais simples e fáceis de serem utilizadas como as medidas do braço e a bioimpedância elétrica $(\mathrm{BIA})^{33}$, até as mais caras e sofisticadas como a tomografia computadorizada, a pletismografia e a dual-energy X-ray absorptiometry (DEXA), considerada padrão-ouro neste tipo de avaliação ${ }^{42}$.

Dentre as medidas do braço, destaca-se a circunferência do braço (CB), que é o parâmetro nutricional antropométrico recomendado pela Organização Mundial da Saúde (OMS) para estimativa da proteína muscular esquelética total $^{43}$, e representa a somatória das áreas constituídas pelo tecido ósseo, muscular e gorduroso do braço. Alguns autores demonstraram sua efetividade em indicar subnutrição, quando comparada às relações $\mathrm{P} / \mathrm{E}$ e IMC/ ${ }^{44,45}$, bem como em predizer mortalidade. Briassoulis et $a l .{ }^{46}$ avaliando 71 crianças gravemente doentes sob ventilação mecânica, encontraram maior mortalidade entre crianças que apresentavam depleção das reservas proteicas e de gordura. Soler-Cataluña et al. ${ }^{47}$ estudando a mortalidade em 96 pacientes do sexo masculino, portadores de doença pulmonar obstrutiva crônica, encontraram na análise multivariada, que valores de área muscular do braço (AMB) inferiores ao percentil (p) 25, foram indicadores independentes de pior 
prognóstico nestes pacientes, até mesmo naqueles que apresentavam peso normal ou sobrepeso. Os autores concluíram que a depleção muscular mensurada pela $A M B$ se mostrou melhor para predizer mortalidade quando comparado ao IMC nestes pacientes. Ravasco et al. ${ }^{44}$ encontraram maior mortalidade entre pacientes avaliados na UTI, com valor de CB inferior ao p5. Nestes pacientes $\mathrm{CB}<\mathrm{p} 15$, foi também capaz de predizer mais complicações.

\section{Bioimpedância elétrica (BIA)}

A BIA é um método simples, portátil, não invasivo e de fácil reprodução para avaliação da composição corporal de pacientes ambulatoriais e internados, em várias situações clínicas; que se baseia na passagem de uma corrente elétrica de baixa intensidade $(800 \mu \mathrm{A})$ e frequência fixa $(50 \mathrm{kHz})$ pelo corpo, mensurando componentes primários - resistência $(R)$, reactância $(X c)$ e ângulo de fase (AF) - e estimando por equações matemáticas: MG, MLG e água corporal total $(\mathrm{ACT})^{48}$. O princípio é de que os tecidos magros são altamente condutores de corrente elétrica, por conter grande quantidade de água e eletrólitos; logo, apresentam baixa resistência. Por outro lado, gordura e osso são pobres condutores, com menor quantidade de fluidos e eletrólitos e maior resistência elétrica. A R é definida como a oposição oferecida pelo corpo à corrente elétrica, primariamente relacionada à quantidade de água presente nos tecidos, e a Xc ou capacitância é o efeito resistivo produzido pelas interfaces dos tecidos e das membranas celulares.

As estimativas feitas pela BIA baseiam-se em dois pressupostos: que a hidratação do tecido é igual em todos os indivíduos e que o corpo se comporta como um cilindro que conduz a corrente elétrica uniformemente. Sendo assim, a BIA parece não ser um bom método para avaliar a composição corporal em 
situações em que esses dois princípios não são válidos, tais como obesidade, pacientes gravemente doentes e/ou com distúrbios de hidratação, ascite e edema $^{49,50}$. Para minimizar este viés, muitas equações de predição que levam em conta valores de peso, altura, sexo e idade foram desenvolvidas para estimar MLG e $\mathrm{ACT}^{51,52}$.

Apesar desta suposta limitação do método na estimativa da composição corporal de grupos específicos, os parâmetros absolutos mensurados pela BIA têm ganhado destaque, não só como indicadores indiretos da condição nutricional, mas também como possível ferramenta de indicação de prognóstico de morbidade e mortalidade em várias situações clínicas como cirurgias, câncer e cuidados intensivos ${ }^{53,54}$. Dentre os parâmetros da BIA, o AF é o mais clinicamente estabelecido e ganhou popularidade na prática clínica, uma vez que vem mostrando grande capacidade de predizer desfechos numa ampla variedade de situações ${ }^{55-59}$

O AF é formado quando uma parte da corrente elétrica a qual o indivíduo é submetido é armazenada na membrana celular, criando assim uma mudança de fase que é quantificada geometricamente pela transformação angular da relação Xc/R $\left[(\mathrm{Xc} / \mathrm{R}) \times\left(180^{\circ} / \mathbb{\Upsilon}\right)\right]^{54}$ (Figura 2). É um marcador obtido diretamente pela análise da BIA (sem a necessidade de utilização de equações), que não sofre interferência da hidratação e que tem sido considerado um marcador de saúde celular, onde valores mais elevados refletem maior conteúdo e integridade de membranas celulares, bem como melhor função celular ${ }^{54,60}$. Este conceito pode ser extrapolado para a condição nutricional, uma vez que todas as propriedades elétricas da membrana celular são influenciadas por mudanças na massa celular, que por sua vez é dependente da taxa metabólica 
e da nutrição ${ }^{61}$. Em indivíduos saudáveis o AF usualmente situa-se entre $5^{\circ} \mathrm{e}$ $7^{\circ}$, e valores acima de $9,5^{\circ}$ podem ser alcançados em atletas ${ }^{62}$. Alguns estudos demonstraram que está diminuído em pacientes com significante perda de peso, confirmando seu status de relevante marcador também de subnutrição, que é caracterizada pelo aumento do fluido extracelular e diminuição da massa celular corporal $^{53,63}$.

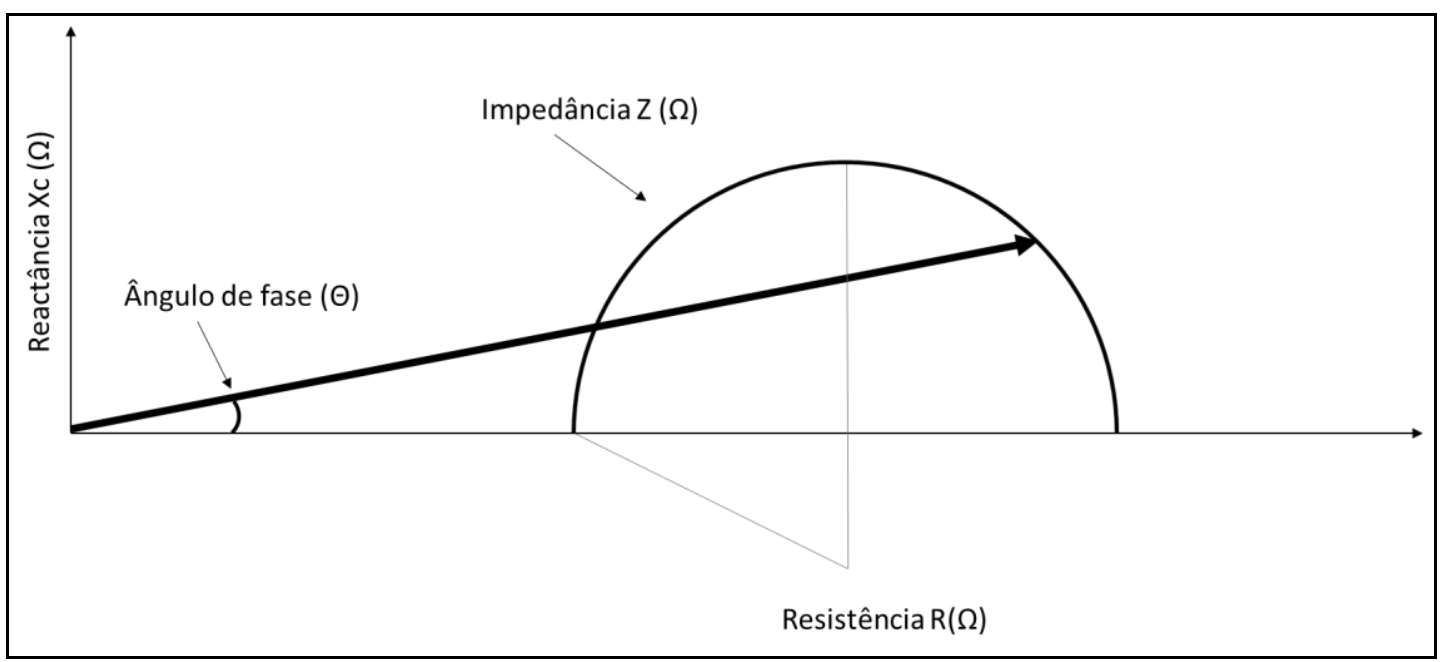

Figura 2 - Demonstração da derivação gráfica do AF (adaptado de Baumgartner et al., $1988^{64}$ )

Recente estudo com 936 adultos gravemente doentes provenientes de 10 centros de nove países, que objetivou determinar a associação entre MLG (representada pelo AF) à admissão na UTI e mortalidade, encontrou valores menores de AF em pacientes que foram a óbito quando comparados aos que sobreviveram; sendo também um fator independente para mortalidade em até 28 dias de internação na população estudada ${ }^{59}$.

Em UTIP, Azevedo et al. ${ }^{65}$ investigaram o comportamento da R e Xc na gravidade dos pacientes, avaliada por injúria pulmonar, sepse e disfunção de 
múltiplos órgãos e sistemas. Entretanto, estudos avaliando a associação de morbimortalidade e AF em crianças gravemente doentes são inexistentes.

Dada a importância da análise da composição corporal como adjunta da avaliação nutricional e como possível indicadora de prognóstico de morbimortalidade em diversas situações clínicas, faz-se importante a realização de estudos que comprovem sua efetividade, especialmente em crianças gravemente doentes, onde os dados ainda são escassos.

O presente estudo tem como hipótese que indicadores de composição corporal são úteis como indicadores de prognóstico em crianças e adolescentes gravemente doentes. 


\section{Objetivos}




\subsection{Geral}

Avaliar a utilidade de marcadores de composição corporal, como potenciais indicadores de prognóstico em crianças e adolescentes gravemente doentes.

\subsection{Específicos}

a) Estabelecer associação entre marcadores de composição corporal (AF e CB) e mortalidade até o $30^{\circ}$ dia na UTIP;

b) Determinar o cutoff de AF associado à mortalidade até $030^{\circ}$ dia em crianças gravemente doentes na UTIP;

c) Estabelecer associação entre marcadores de composição corporal (AF e CB) e morbidade (tempo de internação) na UTIP. 
3 MÉtodos 


\subsection{Descrição da População}

Tratou-se de estudo descritivo e prospectivo. A amostra da população foi sequencial, formada por lactentes, crianças e adolescentes admitidos na UTIP do Instituto da Criança do Hospital das Clínicas da Faculdade de Medicina da Universidade de São Paulo (HC-FMUSP). Esta unidade de 15 leitos de terapia intensiva pediátrica é considerada de nível de atendimento terciário e presta assistência a pacientes de dois meses a 18 anos e 11 meses, sendo a maioria deles portadores de doenças crônicas e frequentemente acompanhados nos ambulatórios de especialidades. No período do estudo, em média $70 \%$ das crianças admitidas nesta unidade faziam uso de ventilação mecânica e $65 \%$ requeriam uma ou mais drogas vasoativas. Com relação às infecções hospitalares, a unidade tem reduzido gradativamente as taxas, estando em conformidade com as recomendações da Coordenadoria de Vigilância em Saúde da Secretaria Municipal Saúde de São Paulo (COVISA).

O tamanho da amostra de conveniência foi calculado com base em haver no mínimo 10 eventos (óbitos) para cada variável prognóstica a ser estudada. Foram observados 36 óbitos provendo assim informações suficientes para incluir as duas variáveis estudadas no modelo ( $\mathrm{CB}$ e $\mathrm{AF})$.

Todos os pais ou responsáveis foram instruídos pelo autor sobre o projeto de pesquisa e assinaram o termo de consentimento livre e esclarecido (TCLE) (Anexo A). 
O estudo foi aprovado pela Comissão de Pesquisa e Ética do Instituto da Criança do HC-FMUSP e na Comissão de Análise de Projetos de Pesquisa da FMUSP (CAPPesq) (Anexo B).

\subsubsection{Critérios de inclusão}

Foram incluídos no estudo todos os lactentes, pré-escolares, escolares e adolescentes, com diagnósticos clínico e cirúrgico, internados na UTIP do Instituto da Criança do HC-FMUSP, no período de um ano.

\subsubsection{Critérios de exclusão}

Foram excluídos do estudo os seguintes grupos de pacientes:

a) recém-nascidos;

b) aqueles que apresentaram óbito precoce, isto é, anterior à avaliação nutricional de admissão (primeiras 24 horas);

c) crianças cujos pais ou responsáveis não concordaram em participar, após exposição dos objetivos da pesquisa e procedimentos aos quais as mesmas seriam submetidas (TCLE).

\subsection{Coleta de dados}

A coleta dos dados foi feita em protocolo próprio criado para o estudo (Anexo C). 


\subsubsection{Avaliação geral}

Constou de dados do prontuário do paciente:

a) número de registro;

b) data de nascimento;

c) idade;

d) sexo;

e) data de admissão;

f) história da moléstia atual;

g) diagnóstico (s) clínico (s).

\subsubsection{Avaliação nutricional antropométrica}

Foi realizada para a caracterização nutricional da população estudada nas primeiras 24 horas de admissão, sempre pelo mesmo pesquisador. Contemplou peso, estatura/comprimento e IMC [(peso $(\mathrm{Kg}) /$ estatura $\left.^{2}(\mathrm{~m})\right]$.

O peso foi aferido em balança digital com escala de precisão devidamente calibrada antes de cada pesagem. Para as crianças menores de dois anos o peso foi mensurado em balança própria com escala de precisão de $5 \mathrm{~g}$ (Filizola Baby®). As crianças maiores de dois anos foram pesadas em pé, de costas para a escala da balança (Welmy RI W 200®, escala de precisão de 100g), com afastamento lateral dos pés, em posição anatômica com o peso do corpo igualmente distribuído entre ambos os pés.

Naquelas crianças com impossibilidade de pesagem (ventilação mecânica, drogas vasoativas), a aferição foi feita com a aplicação da técnica de desconto proposta por Frisancho $(1999)^{66}$. Ou seja, a criança foi pesada no 
colo do avaliador ou do acompanhante, sendo subtraído o peso do mesmo do total obtido. Logo, o peso referido não foi utilizado.

O comprimento foi aferido em crianças até três anos de idade utilizandose 0 antropômetro pediátrico com escala de precisão de $0,1 \mathrm{~cm}$. A medida foi realizada com a criança deitada, em superfície plana e reta, com a cabeça e a planta do pé apoiadas no equipamento.

A estatura foi obtida nas crianças maiores de três anos, com o uso do estadiômetro de madeira com escala de precisão de $0,1 \mathrm{~cm}$. A criança foi mantida em pé, descalçada, com os calcanhares juntos, peso distribuído entre ambos os pés, costas retas, e os braços estendidos ao lado do corpo.

Nas crianças com limitações que impossibilitaram a aferição da estatura por métodos convencionais (ventilação mecânica, medicações vasoativas), a mesma foi obtida utilizando-se a medida da distância entre joelho e tornozelo e as equações propostas por Chumlea et al. ${ }^{67}$ para crianças de 6 a 18 anos [meninas brancas $=43,21+(2,15 \times$ joelho-tornozelo $) ;$ meninas negras $=46,59$ $+(2,02 \times$ joelho-tornozelo $)$; meninos brancos $=40,54+(2,22 \times$ joelhotornozelo); meninos negros $=39,6+(2,18 \times$ joelho-tornozelo $)]$, e comprimento da tíbia proposta por Stevenson et al. ${ }^{68}$ para crianças menores de 2 a 6 anos $[(3,26 \times$ tíbia $)+30,8]$.

O estado nutricional foi classificado pela determinação do escore $Z(Z)$ para IMC/I, tomando como referencial os valores da OMS/2006-2007 ${ }^{69}$. O programa utilizado para os cálculos foi o Who Anthro® (crianças de 1 mês a 5 anos) e Who Anthro Plus ${ }^{\circledR}$ (crianças de 5 a 18 anos e 11 meses) com os valores de normalidade da OMS/2006-2007. 


\subsubsection{Avaliação da composição corporal}

\subsubsection{Antropométrica}

Para esta avaliação foi escolhida a CB (que representa a soma das áreas constituídas pelos tecidos ósseos, muscular e gorduroso do braço), por ser um método simples, barato e facilmente reproduzível, além de ser um bom discriminador de subnutrição em crianças gravemente doentes ${ }^{70}$.

A CB foi avaliada com o auxílio de uma fita métrica com graduação oficial de $0,5 \mathrm{~cm}$ de largura, flexível e inelástica. A criança foi mantida com o braço flexionado em direção ao tórax formando um ângulo de $90^{\circ}$, para a determinação do ponto médio da distância entre o acrômio e o olécrano. A circunferência foi então medida, exatamente neste ponto, com o braço estendido ao longo do corpo (Figura 3). A classificação nutricional foi expressa em $p$, sendo utilizado o referencial proposto por Frisancho (1974) ${ }^{71}$, que contempla todas as faixas etárias: $p \leq 5 ; p$ 5-10; $p$ 10-25; $p$ 25-50; $p$ 50-75; $p$ 75-95 e $p>95$.

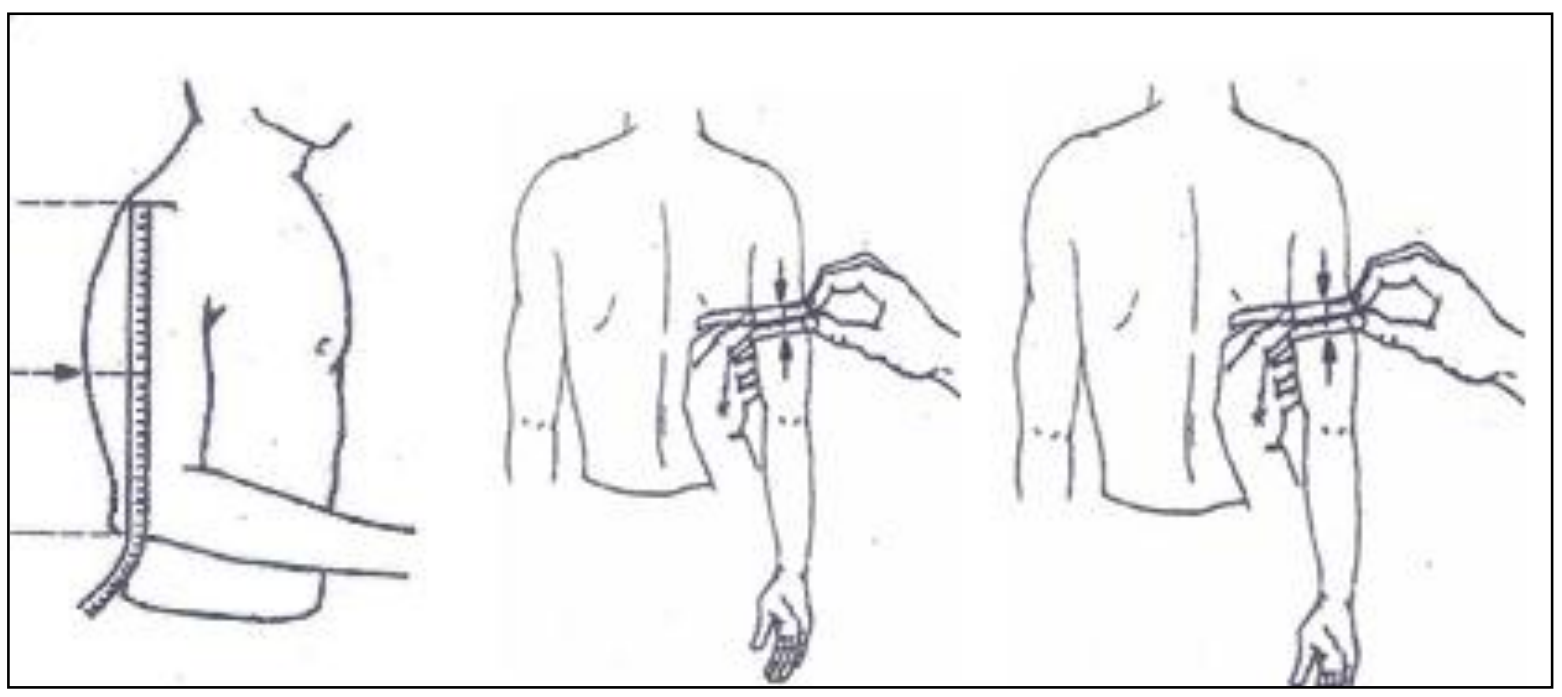

Figura 3 - Mensuração da $\mathrm{CB}^{66}$

Para a análise as crianças foram categorizadas em pCB $\leq 5$ (considerado subnutrição) e pCB > 5 . 


\subsubsection{BIA}

A BIA foi realizada para obtenção dos valores de AF (mensurados diretamente), em todos os pacientes no primeiro dia de admissão usando o modelo tetrapolar Biodynamics 450® (Body Composition Analyzer Biodynamics Corporation, Seattle, WA) com corrente padrão de $800 \mu \mathrm{A}$ e frequência de $50 \mathrm{kHz}$. Após a limpeza da pele com etanol a 70\%, com o paciente em decúbito dorsal com braços e pernas afastados, foram colocados quatro eletrodos adesivos no lado dorsal da mão esquerda, punho esquerdo, pé esquerdo e tornozelo esquerdo (Figura 4). Nos casos de inacessibilidade do lado esquerdo (curativos, acessos), os eletrodos foram colocados no lado direito.
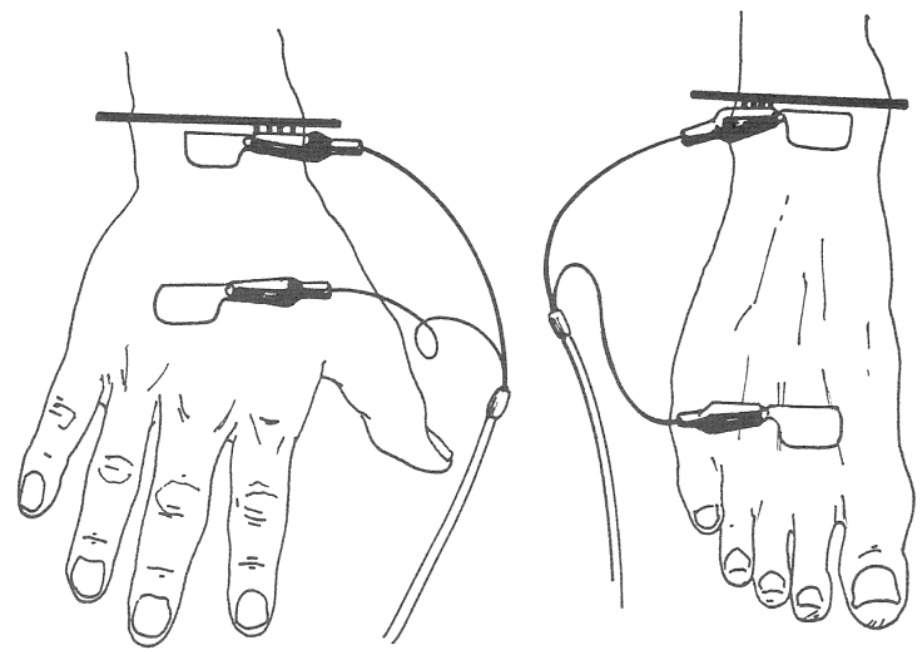

Figura 4 - Colocação dos eletrodos para o exame de $\mathrm{BIA}^{72}$

\subsubsection{Avaliação de risco e gravidade}

Os pacientes admitidos foram avaliados quanto à gravidade aplicando-

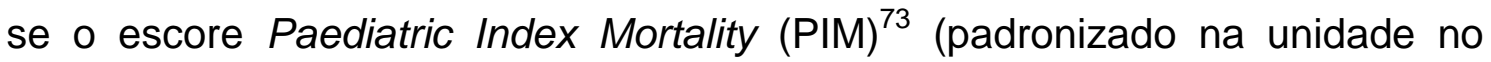
período em que o estudo foi realizado); que utiliza modelo de regressão logarítmico, desenvolvido para predizer o risco de morte em crianças em 
terapia intensiva, baseado em oito variáveis explicativas que são coletadas no momento da admissão.

\subsubsection{Terapia nutricional}

A TN foi iniciada após estabilização clínica e hemodinâmica e seguiu o protocolo estabelecido pela Equipe Multiprofissional de Terapia Nutricional (EMTN) do hospital. As calorias e nutrientes (macronutrientes e micronutrientes) ofertados tanto por via enteral como parenteral foram calculados diariamente. Os incrementos foram feitos de acordo com a tolerância do paciente à $\mathrm{TN}$, visando atender às necessidades nutricionais de cada um individualmente.

\subsubsection{Análise estatística}

Os resultados foram tabulados em planilha do tipo Excel®. Análise descritiva foi utilizada para dados demográficos (idade e sexo), clínicos e antropométricos (Z IMC/I, diagnóstico clínico, gravidade e TN) e variáveis de desfecho (tempo de internação na UTIP e mortalidade). A mediana foi obtida para Z IMC/I, idade (anos), PIM, AF e tempo de internação na UTIP (dias).

Uma curva ROC (Receiver Operating Characteristic) foi construída de acordo com o método de Youden para analisar a associação do AF com a mortalidade em até 30 dias na UTIP, bem como para encontrar o cutoff associado a esta mortalidade. A curva $R O C$ foi analisada com a área sob a curva $(A \cup C)$ e intervalo de confiança (IC) de $95 \%$.

As probabilidades de sobrevivência foram estimadas pelo método de 
Kaplan-Meier e comparadas pelo teste Log-rank. Adicionalmente foi realizada uma regressão de Cox para estimar a taxa de falha das probabilidades. Os modelos de mortalidade incluíram os valores de $\mathrm{AF}$ e $\mathrm{CB}$ e foram ajustados para gravidade, idade, sexo, IMC e PIM.

Para a análise da associação dos indicadores de composição corporal (AF e CB) com tempo de internação, uma curva de Kaplan-Meier foi construída somente com as crianças que sobreviveram, a fim de evitar o viés da influência da mortalidade sobre o tempo de permanência na UTIP. Para a construção da curva, foi estabelecido tempo de internação na unidade de até 40 dias.

Todos os dados foram analisados utilizando o software SPSS 13.0 (IBM Software SPSS, EUA), MedCalc 17.1.0 (MedCalc Software, Bélgica) e o software STATA 11 (StataCorp LLC, EUA).

Os testes foram realizados com nível de significância de 5\%.

A figura 5 resume os principais aspectos metodológicos do estudo. 


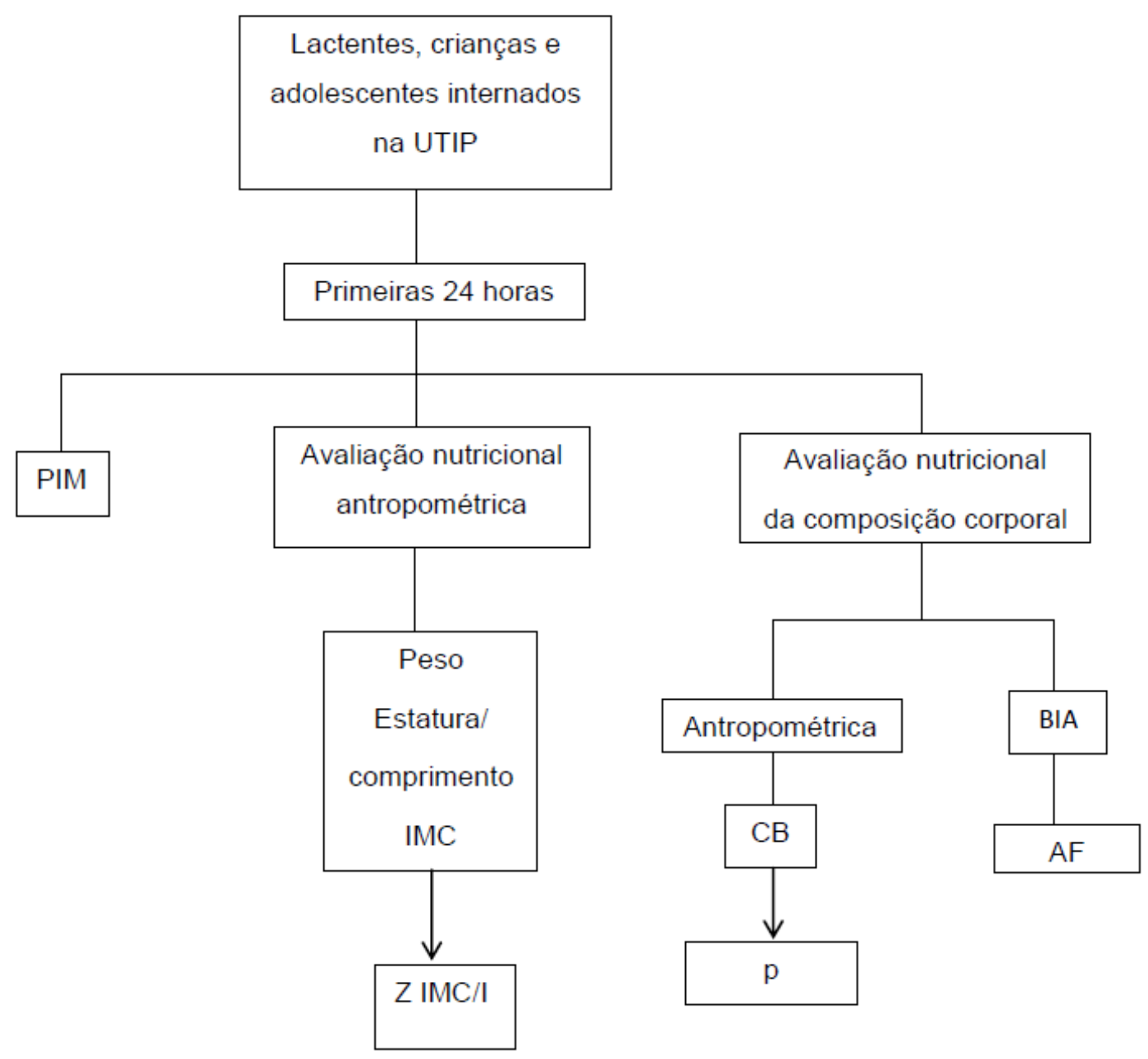

Onde: UTIP = unidade de terapia intensiva pediátrica; PIM = Paediatric Index Mortality; IMC = índice de massa corpórea; $Z \mathrm{IMC} / \mathrm{I}=$ escore $\mathrm{Z}$ de índice de massa corpórea para idade; $\mathrm{CB}=$ circunferência do braço; $\mathrm{p}=$ percentil; $\mathrm{BIA}=$ bioimpedância elétrica; $\mathrm{AF}=$ ângulo de fase

\section{Figura 5 - Metodologia do estudo}


4 Resultados 


\subsection{População}

Com base nos critérios estabelecidos, dentre os 303 pacientes internados no período, foram incluídos inicialmente 271 crianças. Em uma segunda análise foram excluídos 24 (8,8\%), totalizando uma população final de estudo para análise de 247 pacientes (Figura 6). A mediana de idade foi de 4,8 anos e não houve diferenças em relação ao sexo, com 129 meninas (52,2\%) e 118 meninos $(47,8 \%)$. 


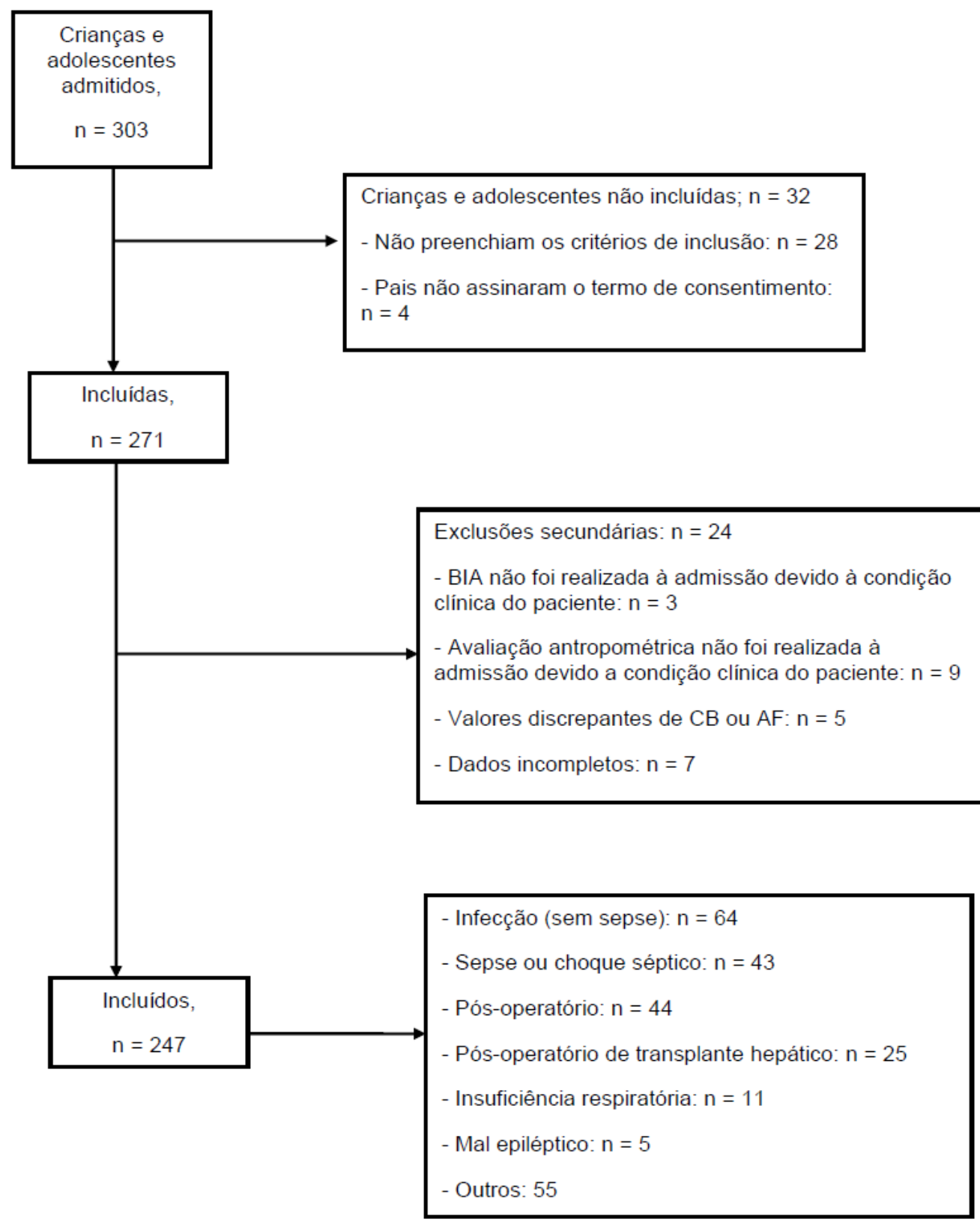

\section{Figura 6 - Fluxograma do estudo}

Durante o período de estudo ocorreram 36 óbitos, correspondendo a $14,6 \%$ dos casos. A mediana do tempo de internação foi de quatro dias e a mediana do PIM foi 2,7.

A oferta média de calorias e proteínas na primeira semana de internação na UTIP (avaliada em 90 pacientes) foi de $77 \mathrm{Kcal} / \mathrm{Kg} / \mathrm{d}$ (mínimo $=17$ 
$\mathrm{Kcal} / \mathrm{Kg} / \mathrm{d}$; máximo $=160 \mathrm{Kcal} / \mathrm{Kg} / \mathrm{d}$ ) e 2,3 g/Kg/d (mínimo = 0,25 g/Kg/d; máximo $=4 \mathrm{~g} / \mathrm{kg} / \mathrm{d}$ ), respectivamente; e a relação de calorias por gramas de nitrogênio foi de 160:1. Houve predomínio de pacientes eutróficos de acordo com Z IMC/I (49,8\%), e quase metade dos pacientes $(49,8 \%)$ apresentou depleção da MLG de acordo com o pCB (abaixo do p5). Os dados referentes ao estado nutricional dos pacientes estão demonstrados na Tabela 1.

Tabela 1 - Estado nutricional de crianças e adolescentes admitidos na UTIP

\begin{tabular}{cc}
\hline Variável & $\mathbf{n}(\%)$ \\
\hline Classificação antropométrica & $37(15,0)$ \\
Subnutrição & $123(49,8)$ \\
Eutrofia & $57(23,0)$ \\
Sobrepeso & $30(12,2)$ \\
Obesidade & \\
pCB & $123(49,8)$ \\
p $\leq 5$ & $109(44,2)$ \\
p 5 - 95 & $15(6,0)$ \\
p $>95$ &
\end{tabular}

\subsection{Sobrevivência}

O cutoff de AF associado à mortalidade em crianças gravemente doentes encontrado na construção da curva $R O C$ foi de $2,8^{\circ}(A U C=0,65$; IC 95\%: 0,58-0,71), com sensibilidade de $37,1 \%$ e especificidade de $86 \%$, como mostra o Gráfico 1. 
Gráfico 1 - Curva ROC de AF vs mortalidade em crianças e adolescentes na UTIP

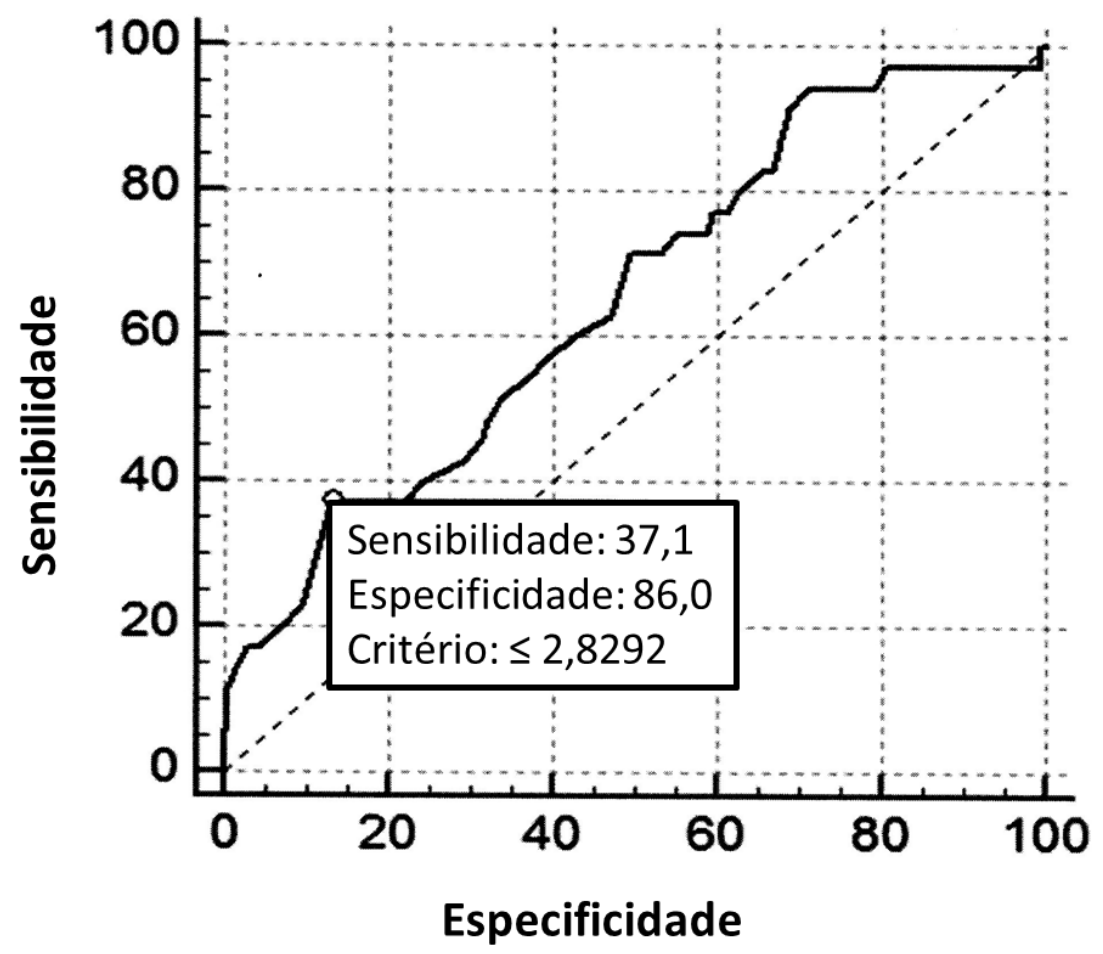

Os pacientes foram estratificados em grupos de acordo com os valores de AF e média do pCB. As curvas de sobrevivência mostraram maior sobrevida nos pacientes com $\mathrm{AF}>2,8^{\circ}$ quando comparados àqueles com $\mathrm{AF} \leq 2,8^{\circ}$; a média foi de 53 dias (IC95\%: 40,5-65,8) e 23 dias (IC95\%: 19,7- 26,8), respectivamente $(p<0,0001)$ (Gráfico 2). Quando analisado o $\mathrm{pCB}$, houve menor sobrevida nos pacientes com $\mathrm{pCB} \leq 5$ em relação aos com $\mathrm{pCB}>5$. A média foi de 26 dias (IC95\%: 24,8-27,9) e 28 dias (IC95\%: 26,4-28,3), respectivamente $(p=0,03)$ (Gráfico 3). 
Gráfico 2 - Curva de sobrevivência Kaplan-Meier para crianças e adolescentes gravemente doentes usando o cutoff de AF obtido pela BIA

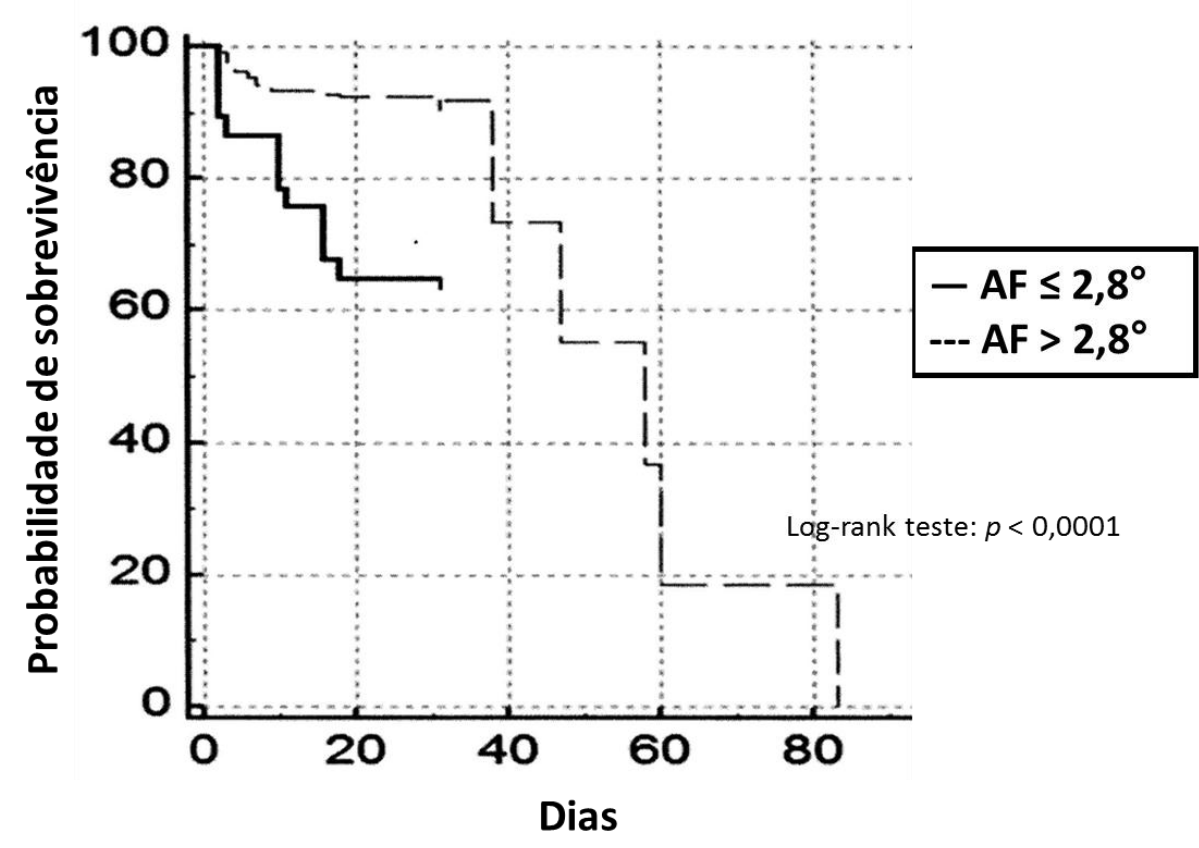

Gráfico 3 - Curva de sobrevivência Kaplan-Meier para crianças e adolescentes gravemente doentes usando o cutoff de pCB

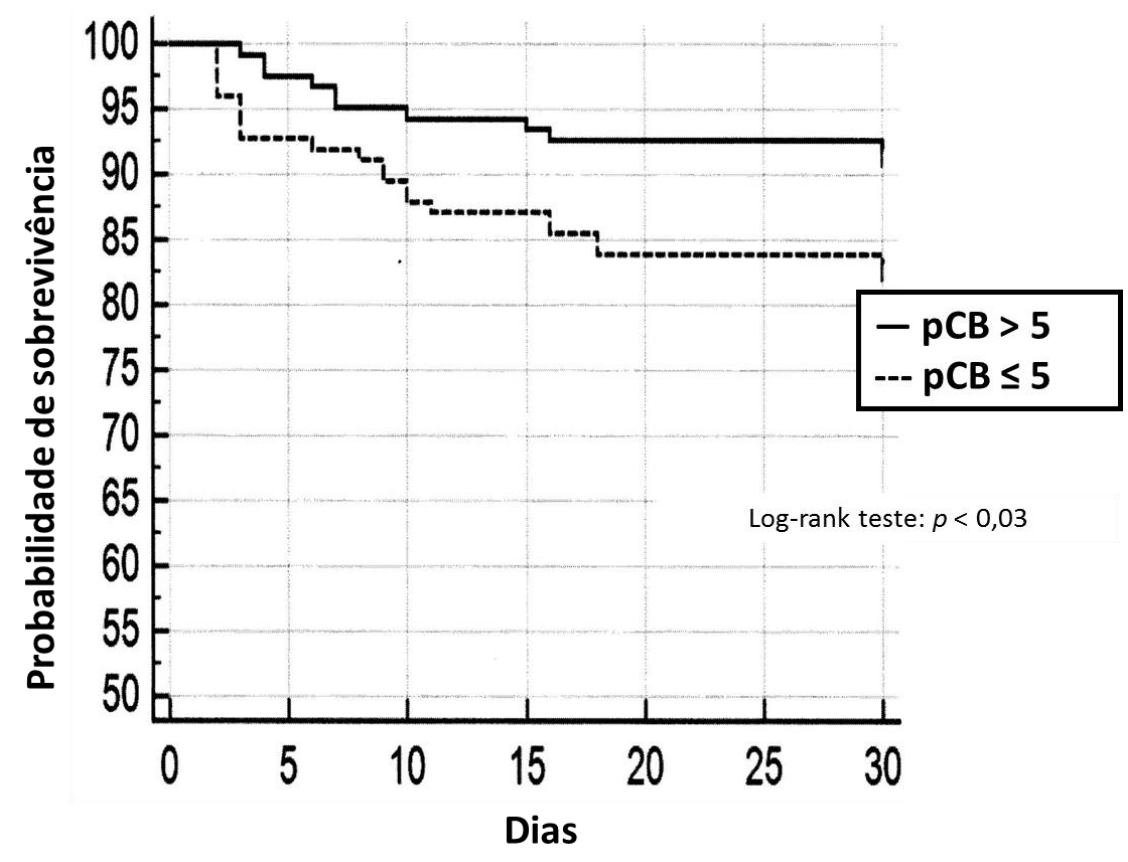


Na regressão de Cox, as variáveis significativamente associadas com a mortalidade em até 30 dias na UTIP foram gravidade da condição clínica (PIM) e AF, como descrito na Tabela 2.

Tabela 2 - Estimativa de mortalidade para crianças e adolescentes gravemente doentes a partir de análise ajustada do modelo de regressão de Cox para AF

\begin{tabular}{cccc}
\hline Variável & $\boldsymbol{H R}$ & IC 95\% & $\boldsymbol{p}$ \\
\hline Idade & 0,99 & $0,98-1,00$ & 0,1337 \\
IMC & 0,96 & $0,80-1,17$ & 0,7475 \\
Sexo & 1,57 & $0,79-3,12$ & 0,1938 \\
AF & 0,66 & $0,48-0,89$ & 0,0073 \\
PIM & 1,03 & $1,02-1,05$ & $<0,0001$ \\
\hline
\end{tabular}

$H R=$ Hazard ratio

A Tabela 3 mostra as variáveis significativamente associadas ao desfecho no modelo para CB.

Tabela 3 - Estimativa de mortalidade para crianças e adolescentes gravemente doentes a partir de análise ajustada do modelo de regressão de Cox para CB

\begin{tabular}{cccc}
\hline Variável & $\boldsymbol{H} \boldsymbol{R}$ & IC 95\% & $\boldsymbol{p}$ \\
\hline Idade & 1,00 & $0,99-1,00$ & 0,7080 \\
IMC & 1,00 & $1,00-1,00$ & 0,9691 \\
Sexo & 1,40 & $0,68-2,86$ & 0,3509 \\
CB & 0,98 & $0,96-0,99$ & 0,0182 \\
PIM & 1,02 & $1,01-1,04$ & $<0,0009$ \\
\hline
\end{tabular}




\subsection{Tempo de internação e indicadores de composição corporal}

Os Gráficos 4 e 5 ilustram as curvas de Kaplan-Meier de associação de AF e $C B$, respectivamente, com tempo de internação. Houve diferença estatisticamente significante entre $A F \leq 2.8^{\circ}$ e $A F>2.8^{\circ}$, bem como entre $p C B$ $\leq 5$ e $\mathrm{pCB}>5$.

Gráfico 4 - Curva de Kaplan-Meier da associação de AF e tempo de internação em crianças e adolescentes gravemente doentes

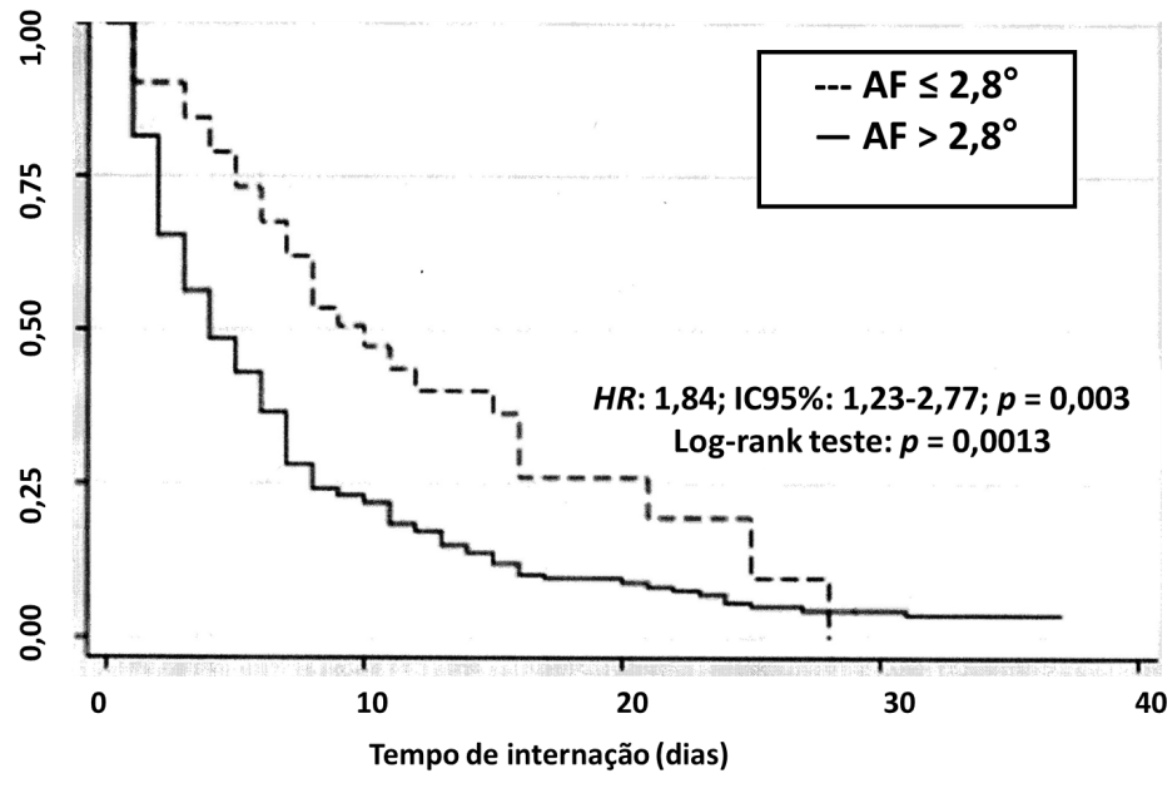


Gráfico 5 - Curva de Kaplan-Meier da associação de CB e tempo de internação em crianças e adolescentes gravemente doentes

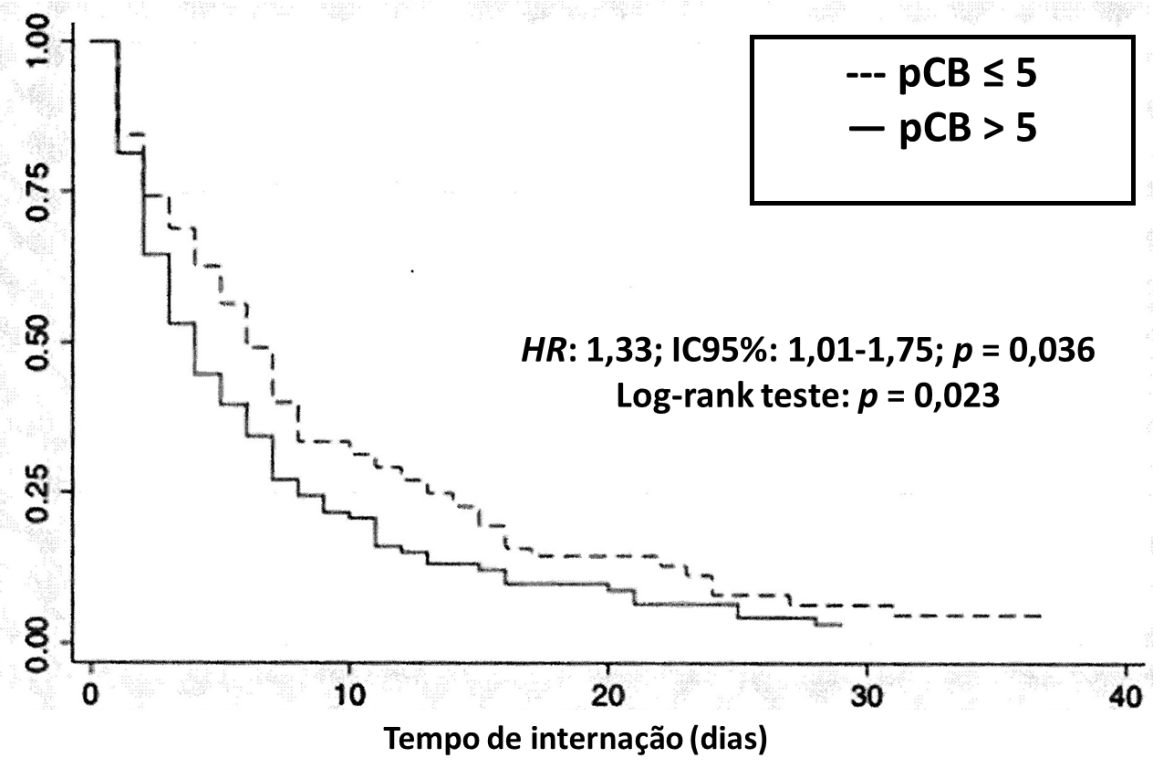

Após ajuste para sexo, idade e gravidade (PIM), a análise da regressão de Cox revelou que as crianças com $\mathrm{AF} \leq 2,8^{\circ}$ tinham probabilidade de permanecerem por mais tempo internadas quando comparadas àquelas com $\mathrm{AF}>2,8^{\circ}(\mathrm{HR}: 1,64 ; p=0,017)$. Os mesmos resultados não foram encontrados quando utilizado o modelo para CB, como demonstrado na Tabela 4.

Tabela 4 - Hazard ratio de tempo de internação em crianças com AF $\leq 2,8^{\circ}$ e $\mathrm{pCB} \leq 5$

\begin{tabular}{l|c|c|c|c}
\hline \multicolumn{1}{c|}{ Variável } & $\begin{array}{c}\text { Análise univariada } \\
\mathrm{HR} \\
(\mathrm{IC} 95 \%)\end{array}$ & $p$ & $\begin{array}{c}\text { Análise multivariada* HR } \\
(\mathrm{IC95} \%)\end{array}$ & $p$ \\
\hline $\mathrm{AF}$ & $1,84(1,23-2,77)$ & 0,0013 & $1,64(1,09-2,47)$ & 0,003 \\
$\mathrm{CB}$ & $1,33(1,01-1,75)$ & 0,0360 & $1,30(0,98-1,71)$ & 0,063 \\
\hline$H R=$ Hazard ratio
\end{tabular}

*ajustada para idade, sexo e PIM 
5 Discussão 
A subnutrição está associada a desfechos desfavoráveis em crianças gravemente doentes. Estudos têm demonstrado que crianças subnutridas em UTIP apresentam aumento do tempo de ventilação mecânica e de internação, além de maior taxa de mortalidade ${ }^{35,74}$.

A subnutrição é comum entre crianças gravemente doentes porque elas apresentam estoques nutricionais reduzidos quando comparadas aos adultos, o que as expõe a maior risco de deterioração da condição nutricional no curso da internação, bem como de morbimortalidade. Sendo assim, a avaliação nutricional precoce, que direciona a TN mais adequada, é de extrema importância no cuidado desses pacientes ${ }^{75}$. Neste estudo de coorte prospectivo de crianças e adolescentes gravemente doentes admitidos em uma UTIP terciária, nós avaliamos a associação entre o estado nutricional na admissão, avaliado por indicadores de composição corporal, e tempo de internação e mortalidade.

Para avaliar o risco de subnutrição e/ou o estado nutricional, vários métodos têm sido utilizados, como as habituais medidas de peso e altura, e os indicadores de composição corporal, que fornecem uma estimativa da gordura corporal e das reservas musculares. O paciente gravemente doente apresenta um intenso catabolismo proteico e mudanças na composição corporal devido à perda de massa muscular e ao rearranjo da ACT. Dessa forma, medidas que definem os compartimentos corporais de forma mais detalhada tornam-se 
importantes, até mesmo como indicadores de prognóstico na avaliação desses pacientes ${ }^{33}$.

Os marcadores de composição corporal, assim como inúmeros outros biomarcadores, têm sido utilizados para mostrar a associação do estado nutricional com desfechos clínicos ${ }^{35,76}$. Estudos têm advogado que o estoque reduzido de massa muscular esquelética é um fator de risco independente para mortalidade em pacientes gravemente doentes, como demonstrado por Weijs et $a l .^{39}$. O estudo avaliou 240 adultos em ventilação mecânica, cujos baixos valores de área muscular (avaliada pela tomografia computadorizada) foram associados a maiores taxas de mortalidade, tanto no sexo feminino $(47,5 \% \mathrm{vs}$ $20 \% ; p=0,008)$ como no masculino $(32,2 \%$ vs $7,5 \% ; p<0,001)$. Os fatores preditivos independentes para mortalidade foram sexo, escore de gravidade e área muscular com OR = 4,3 (IC95\%: 2-9; $p<0,001)$. Moisey et al. ${ }^{77}$ avaliaram o índice de massa muscular, também pela tomografia computadorizada, de 149 idosos e encontraram maior mortalidade nos pacientes considerados sarcopênicos (com índice de massa muscular abaixo do cutoff estabelecido em estudo prévio) quando comparados aos não sarcopênicos (32\% vs 14\%; $p=$ 0,018). Após controle de varáveis como idade, sexo e gravidade clínica, a análise multivariada demonstrou que o aumento do índice de massa muscular estava significativamente associado com a redução da mortalidade $(O R=0,93$; IC95\%: 0,875-0,997; $p=0,004)$.

A antropometria é um método simples, de baixo custo e boa reprodutibilidade, que fornece uma estimativa indireta do estado nutricional e da composição corporal, cuja interpretação precisa requer a utilização de valores de referência para a população estudada ${ }^{47}$. Na avaliação 
antropométrica, as medidas de circunferência e dobras cutâneas refletem os compartimentos de MG e MLG, e auxiliam melhorando a precisão do diagnóstico nutricional, como mostrado por Israels et al. ${ }^{45}$, que avaliando crianças e adolescentes portadoras de câncer pelos indicadores $\mathrm{P} / \mathrm{I}, \mathrm{E} / \mathrm{l}, \mathrm{P} / \mathrm{E}$, CB e dobra cutânea tricipital (DCT), observaram que as medidas do braço foram mais sensíveis para diagnosticar subnutrição nos pacientes. Das 128 crianças estudadas, 59,3\% apresentavam medidas de CMB e DCT abaixo do p5. Em estudo prévio, nós também demonstramos que a CB foi o indicador que melhor discriminou a subnutrição em 256 crianças e adolescentes avaliados em uma UTIP terciária ${ }^{70}$. Estudos como os de Bechard et al..$^{78}$ demonstraram que baixos valores de $\mathrm{Z} \mathrm{IMC/I} \mathrm{constituem} \mathrm{um} \mathrm{indicador} \mathrm{independente} \mathrm{de} \mathrm{mau}$ prognóstico clínico, e que a perda de peso implica em aumento do risco de mortalidade. No entanto, é especulado que os efeitos deletérios atribuídos à subnutrição, na verdade, decorrem da depleção da massa magra e não propriamente da perda de peso corporal. O presente estudo parece corroborar essa ideia, uma vez que a depleção da MLG na admissão na UTIP, representada por $\mathrm{pCB}<5$ (além de baixos valores de $\mathrm{AF}$ ), mostrou associação com a mortalidade em até 30 dias de internação na unidade. Nós não identificamos estudos na literatura descrevendo essa associação em crianças gravemente doentes. Grippa et al..$^{79}$ observaram que a AMB, além do P/l e E/l, foram capazes de predizer a duração da ventilação mecânica em 72 crianças e adolescentes na UTIP, mas associação com mortalidade e tempo de internação não foi estudada. Em adultos, a CMB foi melhor preditora de mortalidade quando comparada ao IMC em pacientes com doença pulmonar obstrutiva crônica, como demonstrado por Soler-Cataluña et al. ${ }^{47}$. Ravasco et 
al. ${ }^{44}$ também observaram maior taxa de mortalidade em adultos gravemente doentes que apresentavam valores de $\mathrm{pCB}<5$.

O IMC é um índice antropométrico amplamente utilizado e válido como marcador nutricional, como mostrado por Bechard et al. ${ }^{78}$; entretanto, pode não evidenciar situações de depleção muscular, uma vez que não discrimina os compartimentos corporais. Nota-se com frequência que indivíduos classificados como eutróficos ou obesos pelo IMC, apresentam redução da massa magra, visto que não necessariamente valores adequados de IMC estão associados à percentis elevados de CB. A maioria dos nossos pacientes foi classificada como eutrófica ou com sobrepeso/obesidade de acordo com o IMC/l. Entretanto, a porcentagem observada de crianças com sobrepeso e obesidade pode ter sido superestimada, uma vez que $90 \%$ das crianças admitidas na UTIP apresentavam doenças crônicas, que frequentemente cursam com subnutrição. Os valores elevados do IMC poderiam ter impacto no $\mathrm{pCB}$, entretanto, isso não foi evidenciado, já que $49,8 \%$ das crianças avaliadas apresentavam MG e MLG reduzidas representadas por valores de CB abaixo do p5. Em estudo prévio, nós mostramos que a maioria dos pacientes foi classificada como eutrófica pelo IMC, mas $58 \%$ deles apresentavam pCB inferior a $5^{70}$. Corroborando os achados acima citados, no presente estudo, a regressão de Cox não mostrou associação entre IMC e mortalidade, mas sim entre $\mathrm{AF}$ e $\mathrm{CB}$ e mortalidade. Esses dados reforçam a importância de complementar a antropometria com a avaliação da composição corporal, não somente para melhorar a precisão do diagnóstico nutricional, mas também para predizer desfechos clínicos. 
A BIA foi introduzida por Lukaski et al. ${ }^{80}$ em 1985 como um método simples de estimar a composição corporal, embora não seja considerada uma boa ferramenta para esta avaliação em situações em que ocorrem alterações da hidratação. Por outro lado, a BIA fornece a mensuração do AF, que é uma medida fácil e rápida, e que reflete indiretamente a $\mathrm{MLG}^{59}$, sem a interferência da água corporal e necessidade da utilização de equações de predição.

O AF está intimamente relacionado à saúde e ao estado nutricional, uma vez que indica tanto a integridade das membranas celulares quanto a distribuição da $A C T^{60,81}$, estando diminuído em pacientes com perda ponderal significativa $^{53}$. Dada sua estreita correlação com o estado nutricional, parece útil na prática clínica, uma vez que tem possibilitado a identificação de pacientes com risco aumentado de subnutrição, bem como reduzido de sobrevivência ${ }^{59}$. Em recente revisão sistemática, dos 455 artigos avaliados com as variáveis de interesse AF e mortalidade/sobrevivência, os autores extraíram 48 estudos, o que permitiu que concluíssem que o AF parece ser um bom indicador de mortalidade em muitas situações clínicas, podendo ser usado para triar os pacientes para este desfecho ${ }^{82}$.

Quando analisamos o AF, observamos associação entre baixos valores (abaixo do cutoff encontrado) e mortalidade em até 30 dias de internação na UTIP, conforme mostrado pela curva de sobrevida e pela regressão de Cox. Além disso, observamos que crianças com os valores reduzidos de AF que não morreram, permaneceram por mais tempo internadas até a alta da UTIP. Køhler et al. ${ }^{83}$ também encontraram associação do AF com o tempo de internação, além da mortalidade, investigando o valor preditivo da composição corporal e da força muscular nos desfechos clínicos de pacientes portadores de 
síndrome do intestino curto que faziam uso de nutrição parenteral prolongada (mais de 12 meses). Ao avaliarem 77 pacientes clinicamente estáveis, encontraram na análise univariada que somente o AF estava associado significativamente com o tempo de internação, ao contrário dos outros índices avaliados: MG, MLG e força muscular. A associação foi confirmada na análise multivariada (coeficiente $=-4,3$ [IC95\%: $-8,6$ a $-0,1] ; p=0,049$ ).

Nosso estudo é um dos primeiros nos últimos anos a explorar o uso de indicadores nutricionais da composição corporal, particularmente o AF, como potenciais indicadores de prognóstico em crianças gravemente doentes, inclusive com a determinação de um cutoff associado à mortalidade nesta população. Nenhum dos 48 estudos que compuseram a revisão sistemática citada $^{82}$ analisou a associação de AF e mortalidade em crianças. Deve-se notar, entretanto, que Azevedo et al. ${ }^{65}$ já haviam investigado o comportamento da $\mathrm{R}$ e Xc em pacientes pediátricos gravemente doentes, avaliado por lesão pulmonar, gravidade da sepse e disfunção de múltiplos órgãos. Em estudo semelhante ao nosso com 196 adultos gravemente doentes, Stapel et al. ${ }^{84}$, encontraram valores mais altos de AF nos indivíduos que sobreviveram quando comparados àqueles que foram à óbito $\left(5,0^{\circ} \pm 1,3^{\circ}\right.$ vs $\left.4,1^{\circ} \pm 1,2^{\circ} ; p<0,001\right)$. Além disso, $\mathrm{AF}<4,8^{\circ}$ (cutoff ideal encontrado pela curva $R O C$ ) foi um fator preditor independente de mortalidade em 90 dias na UTI (OR ajustado para IMC, sexo, idade e escore de gravidade = 3,65; IC95\%: 1,34-9,93; $p=0,011$ ). Thibault et al. ${ }^{59}$ avaliando mais de 900 pacientes adultos obtiveram resultados similares. A MLG mensurada indiretamente pelo AF na admissão na UTI foi associada à mortalidade em até 28 dias, sugerindo que essa medida poderia ser uma das utilizadas para traçar o prognóstico do paciente por ocasião da 
admissão na unidade. Estudos em pediatria ainda são escassos e o cutoff encontrado no presente estudo pode encorajar pesquisas futuras com o AF como indicador de prognóstico também nessa população.

Apesar desses achados, o que contribui para valores reduzidos de AF em condições de doença ainda não está totalmente compreendido. É sabido que idade, sexo e IMC são os principais determinantes do AF em indivíduos saudáveis e que estes valores diminuem com o passar da idade, devido à redução da Xc. Ocorre paralelo a isso, aumento da $\mathrm{R}$ pela diminuição da proporção de ACT, com consequente aumento da MG. Em indivíduos doentes, estudos têm relatado supostos determinantes do $A F$, como desnutrição e inflamação. Em pacientes em hemodiálise, por exemplo, Johansen et al. ${ }^{85}$ mostraram que concentrações mais elevadas de IL-1 estavam associadas com menores valores de AF, que por sua vez, reduziam ao longo do tempo. Stobaüs et al. ${ }^{53}$ observaram que, juntamente com idade e sexo, perda de peso e inflamação foram fatores independentes determinantes do AF em uma grande amostra de pacientes hospitalizados. No atual estudo, além do estado nutricional, a inflamação pode ter sido um importante determinante dos baixos valores de AF encontrados, uma vez que $43 \%$ dos pacientes apresentavam infecção. Além disso, se considerarmos que o AF reduz com a idade, valores mais elevados seriam esperados nestas crianças (com mediana de 4,8 anos), o que não ocorreu, denotando o quão comprometido era seu estado nutricional. Na verdade, o cutoff de AF encontrado nesta população pediátrica foi menor do que os encontrados na literatura: $4,9^{\circ}$ para adultos com cirrose ${ }^{58}$ e $3,4^{\circ 59}$ e $4,8^{\circ 84}$ para adultos gravemente doentes. 
Nosso estudo apresenta limitações como a heterogeneidade da amostra estudada e o fato de os dados terem sido coletados em uma única UTIP terciária. Sendo assim, mais pesquisas são necessárias para determinar se os achados do presente estudo se aplicam a outros centros de terapia intensiva pediátrica. Nós não coletamos outros dados com conhecida utilidade de prognóstico como os marcadores inflamatórios, e não correlacionamos o AF e o pCB com outros desfechos, incluindo morbidades e dias livres de ventilação mecânica.

A associação entre subnutrição e morbimortalidade é conhecida e amplamente documentada na população adulta ${ }^{39,47,56,59,76,86-89}$, entretanto há uma escassez de literatura que demonstre essa associação, avaliada particularmente por indicadores de composição corporal, em crianças doentes e gravemente doentes. Assim, apesar das várias limitações, nosso estudo reforça a importância da avaliação nutricional em crianças gravemente doentes na UTIP, e sugere que a composição corporal pode ser útil não só para o diagnóstico e monitoramento nutricional, mas também como um indicador adicional na estimativa do risco de morte e tempo de internação nessa população. 
6 CONCLUSÕES 
a) Houve associação entre marcadores de composição corporal (AF e CB) e mortalidade até o 30 dia na UTIP;

b) Houve a determinação de um cutoff de AF associado à mortalidade em até 30 dias na UTIP, apropriado para crianças e adolescentes gravemente doentes;

c) Houve associação entre marcadores de composição corporal (AF e CB) e tempo de internação na UTIP;

d) Marcadores de composição corporal podem ser úteis como indicadores de prognóstico em crianças e adolescentes gravemente doentes na UTI. 
7 AnEXos 


\section{Anexo A - Aprovação da CAPPesq}

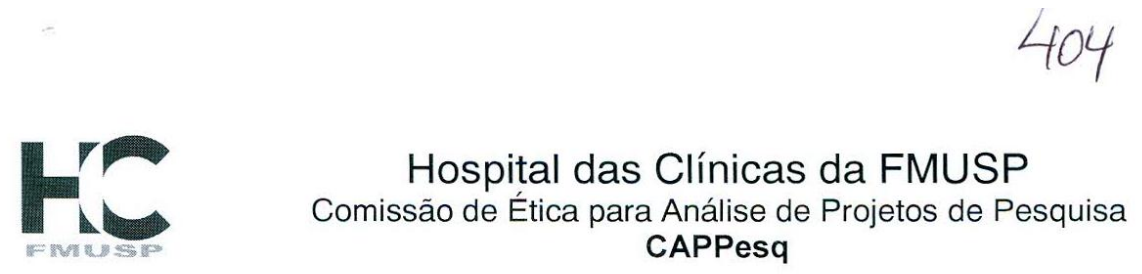

No Protocolo: 0916/04

Título: "Evolução nutricional de crianças internadas em Unidade de Terapia Intensiva Pediátrica"

Pesquisador Responsável: Artur Figueiredo Delgado

Pesquisador Executante: Patrícia Zamberlan dos Santos

Departamento: PEDIATRIA

A Comissão de Ética para Análise de Projetos de Pesquisa CAPPesa da Diretoria Clínica do Hospital das Clínicas da Faculdade de Medicina da Universidade de São Paulo, APROVOU / TOMOU CIÊNCIA na sessão datada de 19/11/2014, do(s) documento(s) abaixo mencionado(s):

- Carta datada de 04.09.14 - Solicitação de troca de pesquisador responsável para Artur Figueiredo Delgado

Em conformidade com a Resolução CNS n 466/12 - cabe ao pesquisador: a) desenvolver o projeto conforme delineado; b) elaborar e apresentar relatórios parciais e final; c)apresentar dados solicitados pelo CEP, a qualquer momento; d) manter em arquivo sob sua guarda, por 5 anos da pesquisa, contendo fichas individuais e todos os demais documentos recomendados pelo CEP; e) encaminhar os resultados para publicação, com os devidos créditos aos pesquisadores associados e ao pessoal técnico participante do projeto; f) justificar perante ao CEP interrupção do projeto ou a não publicação dos resultados.

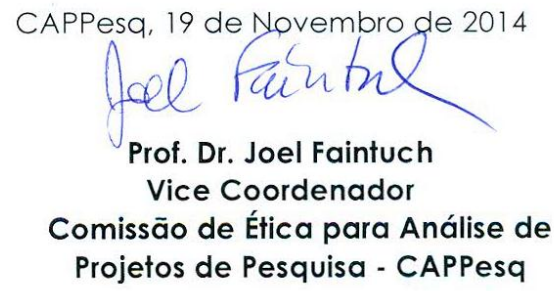

Rua Dr. Ovídio Pires de Campos, 225 - Prédio da Administração - $5^{\circ}$ andar - CEP 05403-010 - São Paulo - SP $55112661-7585$ - $55112661-6442$ ramais: 16,17, 18| marcia.carvalho@hc.fm.usp.br 


\section{4}

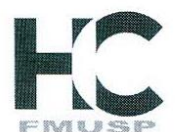

Hospital das Clínicas da FMUSP

Comissão de Ética para Análise de Projetos de Pesquisa CAPPesq

No Protocolo: 0916/04

Título: "Evolução nutricional de crianças internadas em Unidade de Terapia Intensiva Pediátrica"

Pesquisador Responsável: Artur Figueiredo Delgado

Pesquisador Executante: Patrícia Zamberlan dos Santos

Departamento: PEDIATRIA

A Comissão de Ética para Análise de Projetos de Pesquisa CAPPesa da Diretoria Clínica do Hospital das Clínicas da Faculdade de Medicina da Universidade de São Paulo, APROVOU / TOMOU CIÊNCIA na sessão datada de 19/11/2014, do(s) documento(s) abaixo mencionado(s):

- Carta datada de 04.09.14 - Solicitação de troca de pesquisador responsável para Artur Figueiredo Delgado

Em conformidade com a Resolução CNS n 466/12 - cabe ao pesquisador: a) desenvolver o projeto conforme delineado; b) elaborar e apresentar relatórios parciais e final; c)apresentar dados solicitados pelo CEP, a qualquer momento; d) manter em arquivo sob sua guarda, por 5 anos da pesquisa, contendo fichas individuais e todos os demais documentos recomendados pelo CEP; e) encaminhar os resultados para publicação, com os devidos créditos aos pesquisadores associados e ao pessoal técnico participante do projeto; f) justificar perante ao CEP interrupção do projeto ou a não publicação dos resultados.

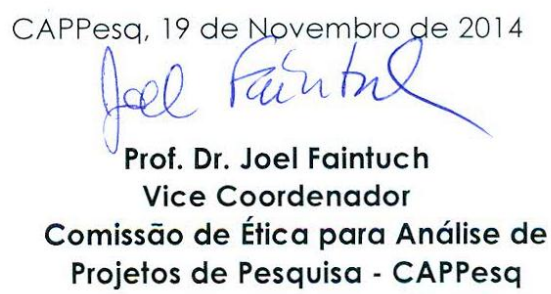

Rua Dr. Ovídio Pires de Campos, 225 - Prédio da Administracão - $5^{\circ}$ andar - CEP 05403-010 - São Paulo - SP $55112661-7585$ - $55112661-6442$ ramais: 16,17,18| marcia.carvalho@hc.fm.usp.br 


\section{Anexo B - Termo de consentimento livre e esclarecido}

\section{HOSPITAL DAS CLÍNICAS \\ DA}

FACULDADE DE MEDICINA DA UNIVERSIDADE DE SÃO PAULO

TERMO DE CONSENTIMENTO LIVRE E ESCLARECIDO

(Instruções para preenchimento no verso)

\section{I - DADOS DE IDENTIFICAÇÃO DO SUJEITO DA PESQUISA OU RESPONSÁVEL LEGAL}

1. NOME DO PACIENTE

DOCUMENTO DE IDENTIDADE № : SEXO : $M \square \quad F \square$

DATA NASCIMENTO:

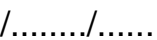

№ …............... АРTO:

ENDEREÇO CIDADE

BAIRRO

CEP:.

TELEFONE: DDD ..)

2.RESPONSÁVEL LEGAL

NATUREZA (grau de parentesco, tutor, curador etc.)

DOCUMENTO DE IDENTIDADE : SEXO: $M \quad \mathrm{~F}$

DATA NASCIMENTO.: ....................

ENDEREÇO:

BAIRRO:

№

$\mathrm{F}$

CEP: CIDADE

APTO:

TELEFONE: DDD ( ..)

\section{II - DADOS SOBRE A PESQUISA CIENTÍFICA}

1. TÍTULO DO PROTOCOLO DE PESQUISA

AVALIAÇÃO NUTRICIONAL DE CRIANÇAS INTERNADAS EM UNIDADE DE TERAPIA INTENSIVA PEDIÁTRICA

PESQUISADOR: ARTUR FIGUEIREDO DELGADO

CARGO/FUNÇÃO: MÉDICO

INSCRIÇÃO CONSELHO REGIONAL №: 52090

UNIDADE DO HCFMUSP: INSTITUTO DA CRIANÇA

3. AVALIAÇÃO DO RISCO DA PESQUISA:

$\begin{array}{lcc}\text { SEM RISCOX } & \text { RISCO MÍNIMO } & \text { RISCO MÉDIO } \\ \text { RISCO BAIXO } & \text { RISCO MAIOR }\end{array}$

(probabilidade de que o indivíduo sofra algum dano como consequência imediata ou tardia do estudo)

4.DURAÇÃO DA PESQUISA : 1 ANO 


\section{III - REGISTRO DAS EXPLICAÇÕES DO PESQUISADOR AO PACIENTE OU SEU REPRESENTANTE LEGAL SOBRE A PESQUISA, CONSIGNANDO:}

1. justificativa e os objetivos da pesquisa: Prezado (a) Senhor (a), como já lhe foi dito, seu filho se encontra num momento mais crítico e grave da doença e precisará de tratamento na UTI (unidade de terapia intensiva), para que seja acompanhado mais de perto. Crianças internadas em UTI, pelo estado clínico mais crítico, podem ter dificuldades na alimentação. Muitas vezes não podem alimentar-se pela boca, e a dieta tem que ser colocada pela veia ou através de um tubo inserido no nariz e que chega até o estômago. Esta dificuldade de alimentação pode prejudicar ou não a parte nutricional da criança. Ou seja, a criança pode chegar nutrida na UTI e durante o tempo de internação, se desnutrir. Ou ao contrário, pode entrar desnutrida e se nutrir. Ou ainda, pode manter-se como chegou. A piora da situação nutricional da criança pode debilitá-la ainda mais, prejudicando sua recuperação e saída da UTI. O objetivo deste estudo do qual seu filho (a) vai participar, é exatamente verificar como as crianças evoluem da parte nutricional (se ganham ou perdem peso, gordura, músculo, água) durante o período de internação na UTI, para que possamos planejar uma alimentação mais apropriada para elas. A verificação da parte nutricional será feita logo na internação, 3 e 7 dias depois desta primeira avaliação, e repetida 1 vez por semana até a alta da UTI (será realizada inclusive na alta). Isto é claro, não quer dizer que seu filho ficará internado por semanas, mas enquanto estiver internado será esta a rotina de avaliação. Para a avaliação serão utilizados dois aparelhos (um chamado adipômetro e outro chamado impedância bioelétrica), que não furam, não machucam, não causam dor nem desconfortos, não emitem radiação e não interferem no funcionamento de outros aparelhos que a criança eventualmente esteja utilizando (monitor, ventilador), exceto marcapasso. A verificação é rápida, no máximo dez minutos por avaliação. Se o (a) senhor (a) não quiser que seu filho (a) participe da pesquisa tem todo o direito de se recusar e ele (a) receberá assim mesmo o tratamento mais adequado para a sua doença. Caso comece na pesquisa e depois deseje desistir também estará garantido para a sua criança o melhor tratamento possível.

2. procedimentos que serão utilizados e propósitos, incluindo a identificação dos procedimentos que são experimentais: Será realizada: pesagem em balança para verificar se a criança ganhou ou perdeu peso; medida da altura/comprimento com régua própria para verificar se a criança cresceu; medida do tamanho (largura) e da dobra do braço com adipômetro para verificar se a criança ganhou ou perdeu gordura e/ou músculo; e impedância bioelétrica com aparelho próprio para também verificar se a criança ganhou ou perdeu músculo e gordura, além de água corporal.

3. desconfortos e riscos esperados: nenhum.

4. benefícios que poderão ser obtidos: conhecimento da situação e evolução da parte nutricional da criança, para um planejamento mais adequado de sua alimentação. Isto contribuirá para uma recuperação clínica mais rápida, com um risco menor de complicações, bem como redução do tempo de internação.

5. procedimentos alternativos que possam ser vantajosos para o indivíduo: nenhum.

\section{IV - ESCLARECIMENTOS DADOS PELO PESQUISADOR SOBRE GARANTIAS DO SUJEITO DA PESQUISA:}


1. acesso a qualquer tempo, às informações sobre procedimentos, riscos e benefícios relacionados à pesquisa, inclusive para dirimir eventuais dúvidas - SIM.

2. liberdade de retirar seu consentimento a qualquer momento e de deixar de participar do estudo, sem que isto traga prejuízo à continuidade da assistência - SIM.

3. salvaguarda da confidencialidade, sigilo e privacidade - SIM.

4. disponibilidade de assistência no HCFMUSP, por eventuais danos à saúde, decorrentes da pesquisa - SIM. Porém a pesquisa não oferece nenhum risco à saúde.

5. viabilidade de indenização por eventuais danos à saúde decorrentes da pesquisa - a pesquisa não oferece nenhum risco à saúde.

\section{INFORMAÇÕES DE NOMES, ENDEREÇOS E TELEFONES DOS RESPONSÁVEIS PELO ACOMPANHAMENTO DA PESQUISA, PARA CONTATO EM CASO DE INTERCORRÊNCIAS CLÍNICAS E REAÇÕES ADVERSAS.}

ENVOLVIDOS NA PESQUISA:

Nome: Patrícia Zamberlan dos Santos e Prof. Dr. Artur Figueiredo Delgado

Endereço: Av. Dr. Enéas de Carvalho Aguiar, 647 CEP: 05403-900 SP.

Fone para contato: $30698558,30698590,97383719$.

\section{OBSERVAÇÕES COMPLEMENTARES:}

\section{VII - CONSENTIMENTO PÓS-ESCLARECIDO}

Declaro que, após convenientemente esclarecido pelo pesquisador e ter entendido o que me foi explicado, consinto em participar do presente Protocolo de Pesquisa.

São Paulo, de de 2

assinatura do sujeito da pesquisa

ou responsável legal assinatura e carimbo do pesquisador 
Anexo C - Instrumento utilizado para a coleta dos dados

PROTOCOLO

1. Identificação

Nome:

RG:

Data de nascimento:

Idade:

Data de internação:

2. História da moléstia atual

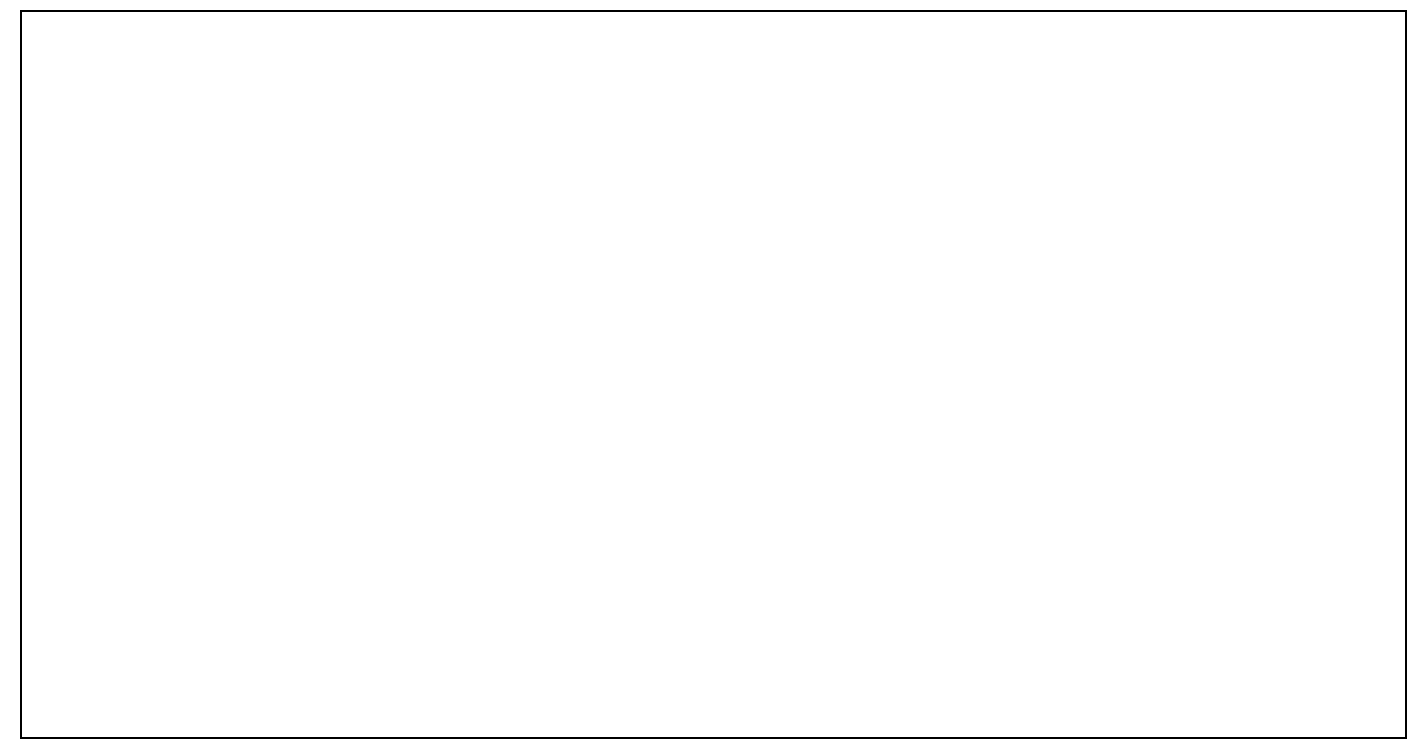

3. Diagnóstico (s) clínico (s)

4. Escore de gravidade (PIM) 


\section{Avaliação nutricional}

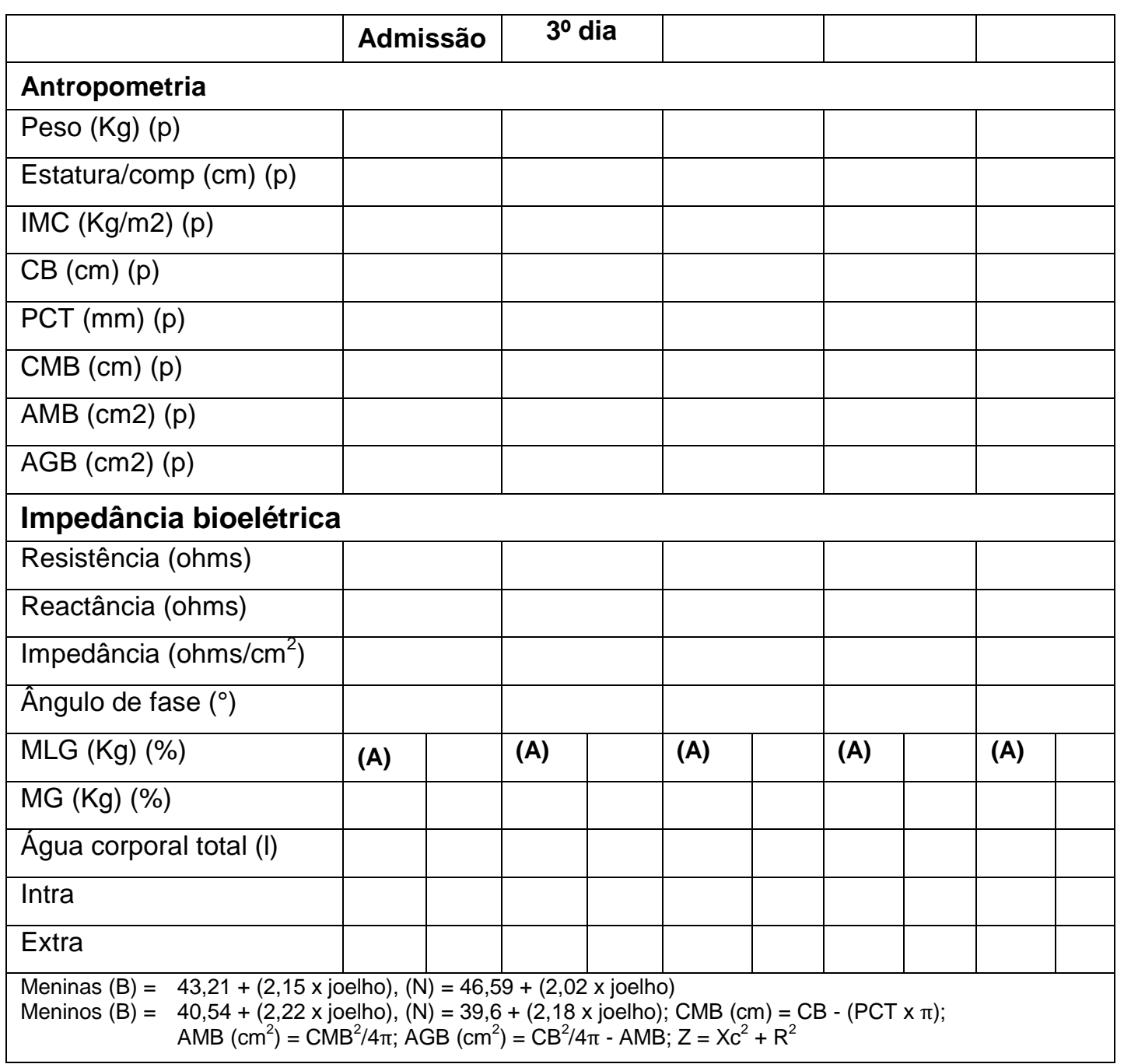


8 REFERÊNCIAS 
1. Mehta NM, Corkins MR, Lyman B, Malone A, Goday PS, Carney LN, Monczka JL, Plogsted SW, Schwenk WF, American Society for Parenteral and Enteral Nutrition Board of Directors. Defining pediatric malnutrition: a paradigm shift toward etiology-related definitions. JPEN J Parenter Enteral Nutr. 2013;37:460-81.

2. Waitzberg DL, Barbosa MRP, Ravacci GR. Desnutrição: definição, prevalência e metabolismo. In: Waitzberg DL. Nutrição oral, enteral e parenteral na prática clínica. 5a ed. São Paulo: Atheneu; 2017. p.533-55.

3. Pawellek I, Dokoupil K, Koletzko B. Prevalence of malnutrition in paediatric hospital patients. Nutrition. 2008;27:72-6.

4. Joosten KFM, Hulst JM. Prevalence of malnutrition in pediatric hospital patients. Curr Opin Pediatr. 2008;20:590-6.

5. Silveira CRM, Mello ED, Carvalho PRA. Evolution of nutritional status of pediatric in patients of a tertiary care general hospital in Brazil. Nutr Hosp. 2008;23:599-606.

6. Huysentruyt K, Alliet P, Muyshont L, Rossignol R, Devreker T, Bontems P, Dejonckheere J, Vandenplas Y, De Schepper J. The STRONG (kids) nutritional screening tool in hospitalized children: a validation study. Nutrition. 2013;29:1356-61.

7. Hecht C, Weber M, Grote V, Daskalou E, Dell'era L, Flynn D, Gerasimidis K, Gottrand F, Hartman C, Hulst J, Joosten K, Karagiozoglou-Lampoudi T, Koetse HA, Kolaček S, Książyk J, Niseteo T, Olszewska K, Pavesi P, Piwowarczyk A, Rousseaux J, Shamir R, Sullivan PB, Szajewska H, Vernon-Roberts A, Koletzko B. Disease 
associated malnutrition correlates with length of hospital stay in children. Clin Nutr. 2015;34:53-9.

8. Pileggi VN, Monteiro JP, Margutti AV, Camelo JS Jr. Prevalence of child malnutrition at a university hospital using the World Health Organization criteria and bioelectrical impedance data. Braz J Med Biol Res. 2016;49(3). pii: S0100-879X2016000300705. Epub 2016 Feb 2.

9. Pollack MM, Wiley JS, Holbrook PR. Early nutritional depletion in critically ill children. Crit Care Med. 1981;9:580-3.

10.Pollack MM, Wiley JS, Kanter R, Holbrook PR. Malnutrition in critically ill infants and children. JPEN J Parenter Enteral Nutr. 1982;6:20-4.

11.Leite HP, Isatugo MK, Sawaki L, Fisberg M. Anthropometric nutritional assessment of critically ill hospitalized children. Rev Paul Med. 1993;111:309-13.

12. Hulst J, Joosten K, Zimmermann L, Hop W, Van Buuren S, Buller H, Tibboel D, van Goudoever J. Malnutrition in critically ill children: from admission to 6 months after discharge. Clin Nutr. 2004;23:223-32.

13. Delgado AF, Okay TS, Leone C, Nichols B, Del-Negro GM, Costa-Vaz FA. Hospital malnutrition and inflammatory response in critically ill children and adolescents admitted to a tertiary intensive care unit. Clinics(São Paulo). 2008;63:357-62.

14.Zamberlan P. O uso de indicadores antropométricos na avaliação nutricional sequencial de crianças e adolescentes gravemente doentes internados em uma Unidade de Terapia Intensiva [dissertação]. São Paulo: Faculdade de Medicina, Universidade de São Paulo; 2009. 108p. 
15. Costa CAD, Tonial CT, Garcia PCR. Association between nutritional status and outcomes in critically-ill pediatric patients: a systematic review. J Pediatr (Rio J). 2016;92:223-9.

16. Correia MITD, Perman MI, Waitzberg DL. Hospital malnutrition in Latin America: a systematic review. Clin Nutr. 2017;36:958-67.

17. Freijer K, van Puffelen E, Joosten KF, Hulst JM, Koopmanschap MA. The costs of disease related malnutrition in hospitalized children. Clin Nutr ESPEN. 2018;23:228-33.

18. Mehta NM, Duggan CP. Nutritional deficiencies during critical illness. Pediatr Clin North Am. 2009;56:1143-60.

19. Agus MS, Jaksic T. Nutritional support of the critically ill child. Curr Opin Pediatr. 2002;14:470-81.

20.Plank LD, Hill GL. Energy balance in critical illness. Proc Nutr Soc. $2003 ; 62: 545-52$.

21. Hasenboehler E, Williams, Leinhase I, Morgan SJ, Smith WR, Moore EE, Stahel PF. Metabolic changes after polytrauma: an imperative for early nutritional support. World J Emerg Surg. 2006;4:1-29.

22. Cruz-Jentoft AJ, Baeyens JP, Bauer JM, Boirie Y, Cederholm T, Landi F, Martin FC, Michel JP, Rolland Y, Schneider SM, Topinková E, Vandewoude M, Zamboni M, European Working Group on Sarcopenia in Older People. Sarcopenia: European consensus on definition and diagnosis: report of the European working group on sarcopenia in older people. Age Ageing. 2010;39:412-23.

23.Kress JP, Hall JB. ICU-acquired weakness and recovery from critical illness. N Engl J Med. 2014;370:1626-35. 
24. Deem S. Intensive-care-unit-acquired muscle weakness. Respir Care. $2006 ; 51: 1042-52$.

25.Schefold JC, Bierbrauer J, Weber-Carstens S. Intensive care unitacquired weakness (ICUAW) and muscle wasting in critically ill patients with severe sepsis and septic shock. J Cachexia Sarcopenia Muscle. 2010;1:147-57.

26. Kizilarslanoglu MC, Kuyumcu ME, Yesil Y, Halil M. Sarcopenia in critically ill patients. J Anesth. 2016;30:884-90.

27.Garnacho-Montero J, Amaya-Villar R, Garcia-Garmendia JL, MadrazoOsuna J, Ortiz-Leyba C. Effect of critical illness polyneuropathy on the withdrawal from mechanical ventilation and the length of stay in septic patients. Crit Care Med. 2005;33:349-54.

28. Ali NA, O'Brien JM Jr, Hoffmann SP, Phillips G, Garland A, Finley JC, Almoosa K, Hejal R, Wolf KM, Lemeshow S, Connors AF Jr, Marsh CB, Midwest Critical Care Consortium. Acquired weakness, handgrip strength, and mortality in critically ill patients. Am J Respir Crit Care Med. 2008;178:261-8.

29. Herridge MS, Cheung AM, Tansey CM, Matte-Martyn A, Diaz-Granados N, Al-Saidi F, Cooper AB, Guest CB, Mazer CD, Mehta S, Stewart TE, Barr A, Cook D, Slutsky AS, Canadian Critical Care Trials Group. Oneyear outcomes in survivors of the acute respiratory distress syndrome. $\mathrm{N}$ Engl J Med. 2003;348:683-93.

30. Chelluri L, Im KA, Belle SH, Schulz R, Rotondi AJ, Donahoe MP, Sirio $\mathrm{CA}$, Mendelsohn AB, Pinsky MR. Long-term mortality and quality of life after prolonged mechanical ventilation. Crit Care Med. 2004;32:61-9. 
31. Aurangzeb B, Whitten KE, Harrison B, Mitchell M, Kepreotes H, Sidler M, Lemberg DA, Day AS. Prevalence of malnutrition and risk of undernutrition in hospitalized children. Clin Nutr. 2012;31:35-40.

32. Kondrup J, Allison SP, Elia M, Vellas B, Plauth M; Educational and Clinical Practice Committee, European Society of Parenteral and Enteral Nutrition (ESPEN). ESPEN Guidelines for Nutrition Screening 2002. Clin Nutr. 2003;22:415-21.

33. Feferbaum R, Delgado AF, Zamberlan P, Leone C. Challenges of nutritional assessment in pediatric ICU. Curr Opin Clin Nutr Metab Care. $2009 ; 12: 245-50$.

34.Wells JCK, Fewtrell MS. Measuring body composition. Arch Dis Child. 2006;91:612-7.

35.Ong C, Han WM, Wong JJ, Lee JH. Nutrition biomarkers and clinical outcomes in critically ill children: a critical appraisal of the literature. Clin Nutr. 2014;33:191-7.

36. Ferrie S, Allman-Farinelli M. Commonly used "nutrition" indicators do not predict outcome in the critically ill: a systematic review. Nutr Clin Pract. 2013;28:463-84.

37. Costa GA, Delgado AF, Ferraro A, Okay TS. Application of the pediatric risk of mortality (PRISM) score and determination of mortality risk factors in a tertiary pediatric intensive care unit. Clinics(Sao Paulo). 2010;65:1087-92.

38.Visser IH, Hazelzet JA, Albers MJ, Verlaat CW, Hogenbirk K, van Woensel JB, van Heerde $M$, van Waardenburg DA, Jansen $N J$, Steyerberg EW. Mortality prediction models for pediatric intensive care: 
Comparison of overall and subgroup specific performance. Intensive Care Med. 2013;39:942-50.

39. Weijs PJ, Looijaard WG, Dekker IM, Stapel SN, Girbes AR, Oudemansvan Straaten HM, Beishuizen A. Low skeletal muscle area is a risk factor for mortality in mechanically ventilated critically ill patients. Crit Care. 2014;18:R12.

40. Rutten EP, Calverley PM, Casaburi R, Agusti A, Bakke P, Celli B, Coxson HO, Crim C, Lomas DA, Macnee W, Miller BE, Rennard SI, Scanlon PD, Silverman EK, Tal-Singer R, Vestbo J, Watkins ML, Wouters EF. Changes in body composition in patients with chronic obstructive pulmonary disease: do they influence patient-related outcomes? Ann Nutr Metab. 2013;63:239-47.

41.Pichard C, Kyle UG, Morabia A, Perrier A, Vermeulen B, Unger P. Nutritional assessment: lean body mass depletion at hospital admission is associated with an increased length of stay. Am $\mathrm{J}$ Clin Nutr. 2004;79:613-8.

42. Woodrow G. Body composition analysis techniques in adult and pediatric patients: how reliable are they? How useful are they clinically? Perit Dial Int. 2007;27(Suppl 2):245-9.

43.Onis M, Yip R, Mei Z. The development of PMB-for-age reference data recommended by a WHO Expert Committee. Bull WHO. 1997;75:11-8.

44. Ravasco P, Camilo ME, Gouveia-Oliveira A, Adam S, Brum G. A critical approach to nutritional assessment in critically ill patients. Clin Nutr. 2002;21:73-7. 
45. Israëls T, Chirambo C, Caron HN, Molyneux EM. Nutritional status at admission of children with cancer in Malawi. Pediatr Blood Cancer. $2008 ; 51: 626-8$.

46. Briassoulis G, Zavras N, Hatzis T. Malnutrition, nutritional índices, and early enteral feeding in critically ill children. Nutrition. 2001;17:548-57.

47.Soler-Catalunã JJ, Sánchez-Sánchez L, Martínez-García MA, Sánchez PR, Salcedo E, Navarro M. Mid-arm muscle area is a better predictor of mortality than body mass index in COPD. Chest. 2005;128:2108-15.

48. Foster KR, Lukasky HC. Whole-body impedance. What does it measure? Am J Clin Nutr. 1996;64(Suppl 1):388-96.

49.Barbosa-Silva MC, Barros AJ, Post CL, Waitzberg DL, Heymsfield SB. Can bioelectrical impedance analysis identify malnutrition in preoperative nutritional assessment? Nutrition. 2003;19:422-6.

50.Barbosa-Silva MCG, Barros AJ. Bioelectrical impedance analysis in clinical practice: a new perspective on its use beyond body composition equations. Curr Opin Clin Nutr Metab Care. 2005;8:311-7.

51.Deurenberg P, van der Kooy K, Leenen R, Weststrate JA, Seidell JC. Sex and age specific prediction formulas for estimating body composition from bioelectric impedance: a cross-validation study. Int $J$ Obes. $1991 ; 15: 17-25$.

52. Deurenberg P, Kusters GSL, Smit HE. Assessment of body composition by bioelectrical impedance in children and young adults is strongly agedependent. Eur J Clin Nutr. 1990;44:261-8. 
53. Stobäus N, Pirlich M, Valentini L, Schulzke JD, Norman K. Determinants of bioelectrical phase angle in disease. Br J Nutr. 2012;107:1217-20.

54. Norman K, Stobäus N, Pirlich M, Bosy-Westphal A. Bioelectrical phase angle and impedance vector analysis - clinical relevance and applicability of impedance parameters. Clin Nutr. 2012;31:854-61.

55. Colín-Ramírez E, Castillo-Martínez L, Orea-Tejeda A, Vázquez-Durán M, Rodríguez AE, Keirns-Davis C. Bioelectrical impedance phase angle as a prognostic marker in chronic heart failure. Nutrition. 2012;28:901-5.

56. Alves FD, Souza GC, Clausell N, Biolo A. Prognostic role of phase angle in hospitalized patients with acute decompensated heart failure. Clin Nutr. 2016;35:1530-4

57.Norman K, Wirth R, Neubauer M, Eckardt R, Stobäus N. The bioimpedance phase angle predicts low muscle strength, impaired quality of life, and increased mortality in old patients with cancer. J Am Med Dir Assoc. 2015;16:173.e17-22.

58. Ruiz-Margáin A, Macías-Rodríguez RU, Duarte-Rojo A, Ríos-Torres SL, Espinosa-Cuevas Á, Torre A. Malnutrition assessed through phase angle and its relation to prognosis in patients with compensated liver cirrhosis: a prospective cohort study. Dig Liver Dis. 2015;47:309-14.

59. Thibault R, Makhlouf AM, Mulliez A, Cristina Gonzalez M, Kekstas G, Kozjek NR, Preiser JC, Rozalen IC, Dadet S, Krznaric Z, Kupczyk K, Tamion F, Cano N, Pichard C, Phase Angle Project Investigators. Fatfree mass at admission predicts 28-day mortality in intensive care unit patients: the international prospective observational study Phase Angle Project. Intensive Care Med. 2016;42:1445-53. 
60. Kyle UG, Bosaeus I, Lorenzo AD, Deurenberg P, Elia M, Gómez JM, Heitmann BL, Kent-Smith L, Melchior JC, Pirlich M, Scharfetter H, Schols AM, Pichard C, Composition of the ESPEN Working Group. Bioelectrical impedance analysis - part I: review of principles and methods. Clin Nutr. 2004;23:1226-46.

61.Nagano M, Suita S, Yamanouchi T. The validity of bioelectrical impedance phase angle for nutritional assessment in children. $J$ Pediatr Surg. 2000;35:1035-9.

62.Barbosa-Silva MC, Barros AJ, Wang J, Heymsfield SB, Pierson RN. Bioelectrical impedance analysis: population reference values for phase angle by age and sex. Am J Clin Nutr. 2005;82:49- 52.

63. Oliveira CM, Kubrusly M, Mota RS, Silva CA, Choukroun G, Oliveira VN. The phase angle and mass body cell as markers of nutritional status in hemodialysis patients. J Ren Nutr. 2010;20:314-20.

64.Baumgartner RN, Chumlea WC, Roche AF. Bioelectric impedance phase angle and body composition. Am J Clin Nutr. 1988;48:16-23.

65. Azevedo ZM, Moore DC, de Matos FA, Fonseca VM, Peixoto MV, Gaspar-Elsas MI, Santinoni E, Dos Anjos LA, Ramos EG. Bioelectrical impedance parameters in critically ill children: importance of reactance and resistance. Clin Nutr. 2013;32:824-9.

66. Frisancho AR. Anthropometric standards for the assesment of growth and nutritional status. USA: University Michigan Press;1999. 
67. Chumlea WC, Guo SS, Steinbaugh ML. Prediction of stature from knee height for black and white adults and children with application to mobilityimpaired or handicapped persons. J Am Diet Assoc. 1994;94:1385-91.

68.Stevenson RD. Use of segmental measures to estimate stature in children with cerebral palsy. Arch Pediatr Adolesc Med. 1995;149:65862.

69.World Health Organization. Multicentre Growth Reference Study Group. WHO Child Growth Standards based on length/height, weight and age. Acta Paediatr. 2006;450:76-85S.

70.Zamberlan P, Delgado AF, Leone C, Feferbaum R, Okay TS. Nutrition therapy in a pediatric intensive care unit: indications, monitoring, and complications. JPEN J Parenter Enteral Nutr. 2011;35:523-9.

71. Frisancho AR. Triceps skin fold and upper arm muscle size norms for assessment of nutritional status. Am J Clin Nutr. 1974;27:1052-8.

72. Heyward VH, Stolarczyk LM. Avaliação da composição corporal aplicada. Barueri: Manole; 2000.

73.Shann F, Pearson G, Slater A, Wilkinson K. Paediatric index of mortality (PIM): a mortality prediction model for children in intensive care. Intensive Care Med. 1997;23:201-7.

74.de Souza Menezes F, Leite HP, Koch Nogueira PC. Malnutrition as an independent predictor of clinical outcome in critically ill children. Nutrition. 2012;28:267-70.

75. Briassoulis G, Briassouli E, Tavladaki T, Ilia S, Fitrolaki DM, Spanaki AM. Unpredictable combination of metabolic and feeding patterns in 
malnourished critically ill children: the malnutrition-energy assessment question. Intensive Care Med. 2014;40:120-2.

76. Leite HP, Rodrigues da Silva AV, de Oliveira Iglesias SB, Koch Nogueira PC. Serum albumin is an independent predictor of clinical outcomes in critically ill children. Pediatr Crit Care Med. 2016;17:e50-7.

77. Moisey L, Mourtzakis M, Cotton BA, Premji T, Heyland DK, Wade CE, Bulger E, Kozar RA, Nutrition and Rehabilitation Investigators Consortium (NUTRIC). Skeletal muscle predicts ventilator-free days, ICU-free days, and mortality in elderly ICU patients. Crit Care. 2013,17:R206.

78.Bechard LJ, Duggan C, Touger-Decker R, Parrott JS, Rothpletz-Puglia P, Byham-Gray, Heyland D, Mehta NM. Nutritional status based on body mass index is associated with morbidity and mortality in mechanically ventilated critically ill children in the PICU. Crit Care Med. 2016;44:15307.

79.Grippa RB, Silva PS, Barbosa E, Bresolin NL, Mehta NM, Moreno YM. Nutritional status as a predictor of duration of mechanical ventilation in critically ill children. Nutrition. 2017;33:91-5.

80.Lukaski HC, Johnson PE, Bolonchuk WW, Lykken Gl. Assessment of fat free mass using bioelectrical impedance measurements of the human body. Am J Clin Nutr. 1985;41:810-7.

81.Kyle UG, Bosaeus I, Lorenzo AD, Deurenberg P, Elia M, Manuel Gómez J, Lilienthal Heitmann B, Kent-Smith L, Melchior JC, Pirlich M, Scharfetter H, M W J Schols A, Pichard C; ESPEN. Bioelectrical 
impedance analysis -part II: utilization in clinical practice. Clin Nutr. 2004;23:1430-53.

82.Garlini LM, Alves FD, Ceretta LB, Perry IS, Souza GC, Clausell NO. Phase angle and mortality: a systematic review. Eur J Clin Nutr. 2018 Apr 26. [Epub ahead of print]

83. Køhler M, Olesen SS, Rasmussen HH. Body composition predicts clinical outcome in patients with intestinal failure on long-term home parenteral nutrition. Clin Nutr ESPEN. 2018;28:193-200.

84. Stapel SN, Looijaard WGPM, Dekker IM, Girbes ARJ, Weijs PJM, Oudemans-van Straaten HM. Bioelectrical impedance analysis-derived phase angle at admission as a predictor of 90-day mortality in intensive care patients. Eur J Clin Nutr. 2018;72:1019-25.

85.Johansen KL, Kaysen GA, Young BS, Hung AM, da Silva M, Chertow GM. Longitudinal study of nutritional status, body composition, and physical function in hemodialysis patients. Am J Clin Nutr. 2003;77:8426.

86. Pérez Camargo DA, Allende Pérez SR, Rivera Franco MM, Álvarez Licona NE, Urbalejo Ceniceros VI, Figueroa Baldenegro LE. Phase angle of bioelectrical impedance analysis as prognostic factor in palliative care patients at the National Cancer Institute in Mexico. Nutr Cancer. 2017;69:601-6.

87. Belarmino G, Gonzalez MC, Torrinhas RS, Sala P, Andraus W, D'Albuquerque LA, Pereira RM, Caparbo VF, Ravacci GR, Damiani L, Heymsfield SB, Waitzberg DL. Phase angle obtained by bioelectrical 
impedance analysis independently predicts mortality in patients with cirrhosis. World J Hepatol. 2017;9:401-8.

88. Mauricio SF, Ribeiro HS, Correia MI. Nutritional status parameters as risk factors for mortality in cancer patients. Nutr Cancer. 2016;68:949-57.

89. Norman K, Wirth R, Neubauer M, Eckardt R, Stobäus N. The bioimpedance phase angle predicts low muscle strength, impaired quality of life, and increased mortality in old patients with cancer. J Am Med Dir Assoc. 2015;16:173.e17-e22. 